\title{
Evaluating the Bender Visual Motor Gestalt Test II as a Diagnostic Screening Instrument Among Clinically Referred Children and Adolescents
}

Linda R. Marnic

West Virginia University

Follow this and additional works at: https://researchrepository.wvu.edu/etd

\section{Recommended Citation}

Marnic, Linda R., "Evaluating the Bender Visual Motor Gestalt Test II as a Diagnostic Screening Instrument Among Clinically Referred Children and Adolescents" (2010). Graduate Theses, Dissertations, and Problem Reports. 3162.

https://researchrepository.wvu.edu/etd/3162

This Dissertation is protected by copyright and/or related rights. It has been brought to you by the The Research Repository @ WVU with permission from the rights-holder(s). You are free to use this Dissertation in any way that is permitted by the copyright and related rights legislation that applies to your use. For other uses you must obtain permission from the rights-holder(s) directly, unless additional rights are indicated by a Creative Commons license in the record and/ or on the work itself. This Dissertation has been accepted for inclusion in WVU Graduate Theses, Dissertations, and Problem Reports collection by an authorized administrator of The Research Repository @ WVU.

For more information, please contact researchrepository@mail.wvu.edu. 
Evaluating the Bender Visual Motor Gestalt Test II as a Diagnostic Screening Instrument Among Clinically Referred Children and Adolescents

\author{
Linda R. Marnic
}

Dissertation submitted to the College of Human Resources and Education

at West Virginia University

in partial fulfillment of the requirements

for the degree of

\title{
DOCTOR OF PHILOSOPHY
}

In

Counseling Psychology

\author{
James Bartee, Ph.D., Chair \\ Jeffrey Daniels, Ph.D \\ Margaret Glenn, Ed.D. \\ Richard Walls, Ph.D. \\ Eric Youngstrom, Ph.D.
}
Department of Counseling, Rehabilitation Counseling, and Counseling Psychology
Morgantown, West Virginia
2010

Key Words: Bender Gestalt II, Koppitz 2, KSADS-PL+, CBCL, Children and Adolescents, Clinical Utility. 


\title{
ABSTRACT \\ EVALUATING THE BENDER VISUAL MOTOR GESTALT TEST II AS A DIAGNOSTIC SCREENING INSTRUMENT AMONG CLINICALLY REFERRED CHILDREN AND ADOLESCENTS
}

\author{
LINDA R. MARNIC
}

This research was designed to investigate the diagnostic utility of the Bender Gestalt II (BGII) test using the Bender Global Scoring System (BGSS) and the Koppitz 2 scoring systems. The scores from these two systems were correlated with scores derived from the Kiddie Schedule for Affective Disorders and Schizophrenia for School Aged Children, Present and Lifetime Edition (KSADS-PL+), a semistructured interview along with Longitudinal Evaluation of all Available Data (LEAD) and the Child Behavior Checklist (CBCL). Of the 115 children and adolescents who initially participated in the study to assess the validity of the Bender Visual Motor Gestalt II test as a screening instrument in psychological decision making, 75 completed all protocols and the relevant data were entered into the subsequent analysis. A correlational design was employed, and a post hoc test was used to incorporate Receiver Operating Characteristic (ROC) analysis into the findings. Results from both the Bender Gestalt II Global Scoring System and Koppitz 2 scoring systems showed moderate correlations with results from the CBCL on the symptom categories of aggressive behaviors, depressed behaviors, and attention deficit hyperactivity disorder (ADHD) in children and adolescents. However, the Koppitz 2 Emotional Indicators scoring measure did not accurately discriminate for the presence or absence of psychopathology. The Koppitz 2 Total Error score was found to be modestly correlated with receiving a diagnosis of ADHD. None of the other diagnoses based on results from KSADS-PL+ with LEAD showed any significant correlations with the Koppitz 2 Total Error Score. Adding Receiver Operating Characteristic analysis for sensitivity and specificity, improved the diagnostic likelihood ratio from $50 \%$ to $66 \%$ for ADHD diagnosis using the Koppitz 2 Emotional Indicators. The main hypothesis that the Bender Gestalt II would improve diagnostic accuracy of psychopathology was not supported. The unexpected finding that the BGII is useful in diagnosing ADHD indicates a possible direction for future research. 


\section{Acknowledgments}

I would like to thank all the people who have been instrumental in the completion of this dissertation. I would like to thank Eric Youngstrom, who believes in promoting the field and cultivating the next generation of psychologists. His positive demeanor and endless energy is a benefit not only to the areas of research and academia but to each student under his tutelage. I cannot thank enough, Margalit Persing, Andrew Freeman, and Dr. James Bartee for their editorial work and numerous reviews that made this paper readable. Also, I am grateful to the whole Applewood family who made my years in Cleveland truly wonderful. My inspiration has been the various married working mothers in the field of psychology. They have shown me that it's not impossible to achieve your dreams, impact the lives of others, and still have enough left to be active, loving mothers to wonderful children and partners to the men in their lives. I have found from their examples, the more you give the more you have to give. Thank you.

I would like to acknowledge my family and friends. Stephen, my partner in this life, your love and patience is my strength. You have given the most valuable gift in my life: our children. My life is chaotic and difficult with work, kids, activities, and finding time to write: however, you have stood by me, honored me, and that means so much. Thank you.

I would like to thank all the clients I have seen over the years. Without them I would have never started this endeavor and maintained the belief that I make a difference. I know that I have learned from them as much as they from me. I realize that 
this paper is truly a gift from God, and I could not achieve anything without His help. When I thought all was lost, and I couldn't take another disappointment, I found strength through prayer. I also thank the many who encouraged me to finish and were there for me. Thank you. I am a better person for all the relationships I have had or will have in my life. They have shown me the person I can be and what I am capable of doing. I thank you all. I am truly blessed. If it were not for them then I would not have become me. I am grateful and indebted to all of you. 


\section{Table of Contents}

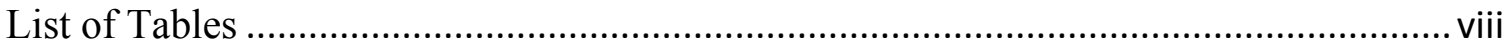

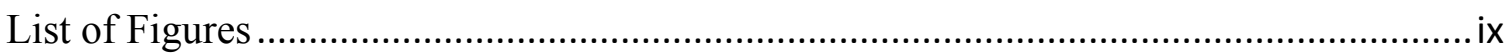

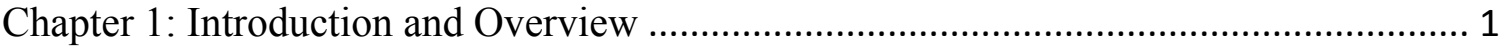

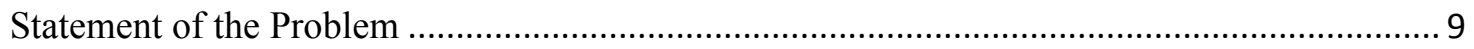

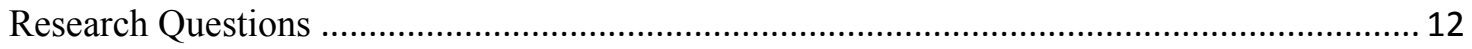

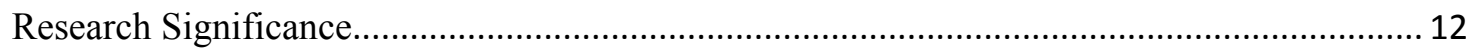

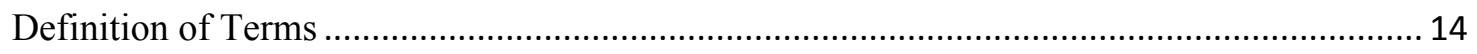

Chapter 2: Review of Selected Literature .......................................................... 21

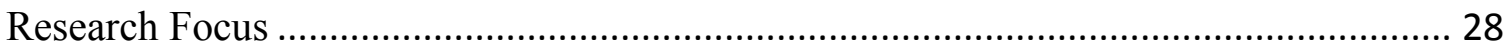

The Bender Visual Motor Gestalt Test ................................................................... 30

Current Use of the Bender Visual Motor Gestalt-Second Edition .......................................... 33

The Koppitz 2 and the Koppitz Developmental Scoring System ............................................ 35

Kiddie Schedule for Affective Disorders and Schizophrenia for School-Age Children ........... 37

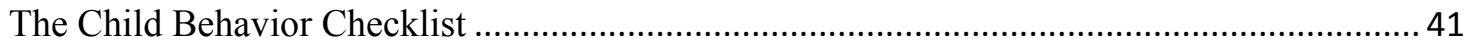

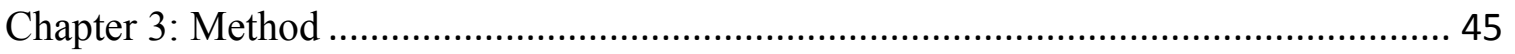

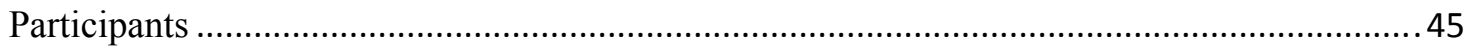


The Bender Visual Motor Gestalt II- Global Scoring System.

The Kiddie Schedule for Affective Disorders and Schizophrenia for School-Age Children (K-SADS).

The Child Behavior Checklists, (CBCL) and Youth Self Report Form (YSR).

Examiners 54

Procedure. .54

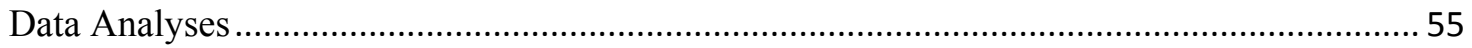

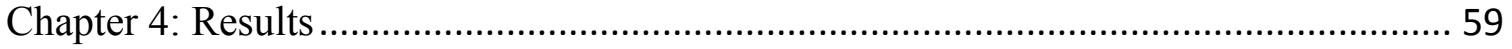

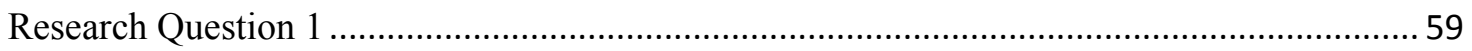





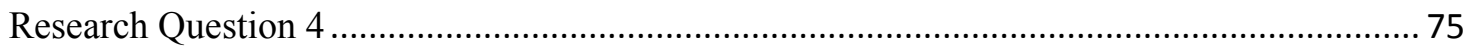

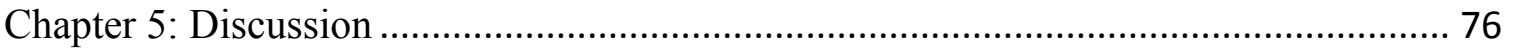

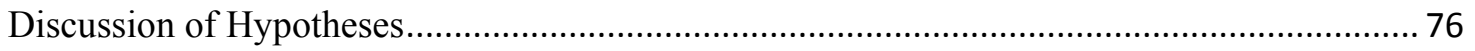

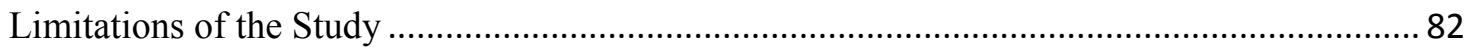

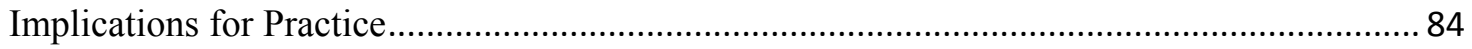

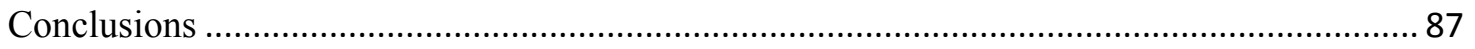

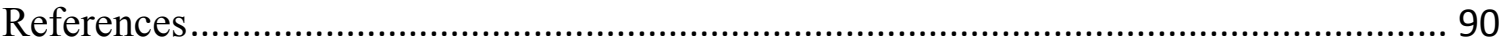

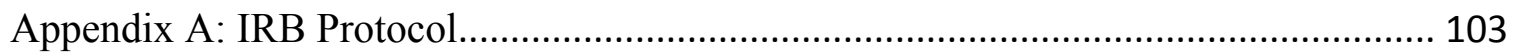

Appendix B: CREC Program Notice of Certification............................................... 108 
Appendix C: Administration Manual for ABACAB .................................................. 109

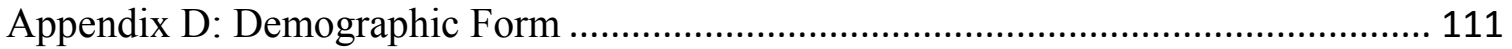



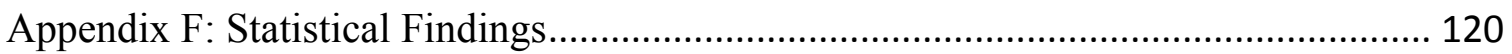

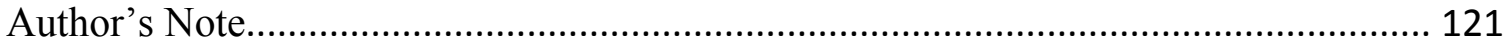




\section{List of Tables}

Table 1 Pearson Product-Moment Correlation Matrix Between the BGSS Copy and

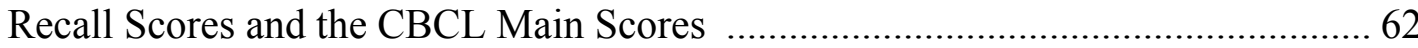

Table 2 Pearson Correlational Matrix of the BGSS Scores and the CBCL Syndrome

Scores

Table 3 Pearson Correlational Matrix Comparing BGSS and CBCL Diagnostic

Scores

Table 4 Pearson Correlational Matrix Comparing CBCL and Koppitz 2 Scores 66

Table 5 Pearson Correlation Matrix Comparing Koppitz 2 Total Score, Total Emotional Indicators, and Visual Motor Index to the CBCL Syndrome Scales 67

Table 6 Pearson Correlation Matrix Comparing the BGII, BGSS and Koppitz2 Scores and KSADS-PL+ Symptomology.

Table 7 Pearson Product-Moment Correlations of the Koppitz 2 Scores and KSADS-PL+

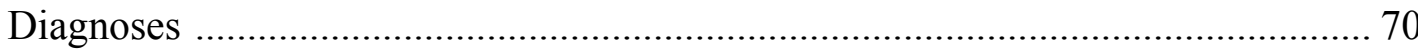




\section{List of Figures}

Figure 1. ROC of errors made using the Koppitz 2 scoring system to predict................ 74 


\section{Chapter 1: Introduction and Overview}

In an era of increased emphasis on accountability and outcomes, psychologists are continually pushed to become more accurate in diagnosing and treating clients while balancing the cost of providing services. The task of developing more efficacious diagnostic and treatment protocols is indeed a challenging one in this time of managed care and limited client contact in part due to insurance considerations and heavy caseloads. Finding valid diagnostic measures that are inexpensive, easy to administer, reliable, and that serve multiple purposes would be beneficial to all. This research was used to investigate the use of the Bender Visual Motor Gestalt II (BGII) as a possible diagnostic tool to add to clinical protocols that assist in initial diagnostic decision making for children and adolescents.

The field of psychology is faced with many challenges. Mental-health statistics for children in the United States are very disturbing. More than 400,000 children are in therapy for treatment of a diagnosed mental illness (Kamphaus, Petoskey, \& Rowe, 2000). Additionally, over 5 million children have received psychoeducational evaluations in public schools to assess for learning, behavior, and information processing problems (Kamphaus et al., 2000). Astoundingly, this number does not include those children who were evaluated outside of the school system for various mental-health or neurological problems. It has been reported by Tolan and Dodge (2005) that over two thirds of the children and adolescents who receive mental-health services have been previously treated, and over three quarters of adult clients in treatment report that their problems 
began in their childhood years. These staggering numbers further highlight the need for increased accuracy and more efficient diagnostic protocols in practice. The importance of early identification of mental-health disorders and the need for effective forms of treatment have been the focus of the American Psychological Association for several years (Tolan \& Dodge, 2005). However, it is important to note that effective treatment relies on the ability of the treating clinician to provide an accurate diagnostic profile of the individual.

Diagnosis is a cornerstone of the practice of counseling psychology. Nowhere is this more evident than in the work with children and adolescents. Not only is the formulation of a diagnosis the first step in treatment planning, but it also lays the foundation upon which all future therapeutic work is built. The Diagnostic and Statistical Manual, 4th edition Text Revision (DSM-IV-TR) is the current manual on which all clinical diagnostic decisions are based and is considered to be the most comprehensive psychological diagnostic manual to date (American Psychiatric Association, 2000). Use of the $D S M-I V$-TR has been credited with an increase in appropriate diagnoses and early intervention in significant childhood mental illnesses. This in turn has led to improved childhood outcomes in autism spectrum disorders, attention deficit hyperactivity disorder, childhood depression, and bipolar disorder (American Psychiatric Association, 2000; Charman \& Baird, 2002; Lipovsky, Finch, \& Belter, 1989; Valderhaug \& Ivansson, 2005). However, one of the criticisms of reliance on the $D S M-I V-T R$ is that the manual allows too much overlap among different diagnoses (DeClercq, DeFruyt, Van Leewen, \& 
Mervielde, 2006). Questions have also been raised about the meaning of disorders categorized as adult disorders with consideration of a childhood onset, leading to further overlap and difficulty among professionals with regard to treatment venues (Sourander et al., 2005).

Psychologists have long sought quick, inexpensive, and empirically sound measures that would assess pathology while giving maximum information with minimum time spent scoring. The original Bender Visual Motor Gestalt (OBG) Test has always been one such quick and direct measure of a child's ability to perform visual motor integration tasks. The OBG has a history of meeting these needs by being fast, easy to administer, and capable of assessing multiple areas of client ability. It has been used by clinicians as a standard measure in psychological batteries for more than 60 years. Before any further detailed discussion of the revised Bender and its use in this research, a brief discussion of the history of this significant test is in order.

The studies of children using direct measures, rather than the use of questionnaires, have been debated for many years. German psychologists Brentano (1838-1917) and Stumpf (1848-1936) supported the study of children and advocated experimental studies based on internal cognitive processes rather than the questionnaire approach pioneered by Hall (1844-1924) and early functionalists in the United States (Hothersall, 1995).

The use of drawing as a medium for the measure of visual- motor integration ability has been. The original Bender-Gestalt Test (OBG) titled The Bender Visual Motor 
Gestalt Test (Bender, 1938), was composed of drawings to be visually perceived and reproduced by patients and was developed by Wertheimer (1880-1943) in his perceptual psychology experiments and later formalized by Bender (1897-1987). Wertheimer was one of the original Gestalt theorists whose theoretical works were cut short by World War II and his flight from Nazi Germany. Although he came to the United States from Germany, he did little to advance Gestalt theory in the United States in the years after the war (Hothersall, 1995). Bender (1938), a psychologist working in New York, further experimented with the figures by using the drawings with both her child and adult patients. The resulting cards which Bender developed were slightly different than the visual motor patterns of the originals by Wertheimer. The resultant client drawings were considered an integration of the discrete internal processes of the drawer (Bender, 1938). Based on Gestalt theory, the resultant drawing represents more than visual and motor associations within the physical body. The drawings reflect association and cognitive complexity within the individual. Individual variables that may affect the accuracy of a drawing include age, physical and emotional development, as well as individual mental and emotional states (Bender, 1938).

According to Bender (1938), developmental maturation of drawing is an ongoing process that follows sequential stages, incorporating motor development from gross to fine motor, visual imagery, and perceptual awareness. Thus, a beginning scribble becomes more circular. Circles become loops and a tendency from vertical to horizontal movements occurs, and then finally, dimensional awareness emerges in the drawing. 
These factors are all components of the maturational process that leads to the more intricate representations found within mature representational drawings which then represent a completed integration of internal processes. A deviation within this developmental or maturational process would obviously lead to a disintegration of the original representation. Psychology has found this process helpful in identifying those individuals who have not yet matured, were delayed in visual motor perception, or who were once matured, yet for various reasons may be losing such integration faculties.

The original Bender test (OBG) consists of nine figures on separate 3 x 5 cards. There have been many research articles that have utilized the Bender as a criterion in developmental processing, perceptual motor skills, and neurological intactness (Brannigan \& Decker, 2003). Horn and O’Donnell (1984) researched the early identification of learning disabilities and found that the OBG was effective in identifying those children classified as learning disabled and those with low achievement. Based on original theory, the cards were also used as a direct measure of the underlying emotional state in those thought to be of normal development and as a personality assessment to assess internal motives (Hutt, 1985). The OBG has also been used as a test for emotional problems and a personality test. The OBG was found to be useful in comparing impulsive adolescents to those without impulse difficulties (Oas, 1984). Oas (1984) found that adolescents with impulsivity disorders were significantly different from those designated as nonimpulsive on the Matching Familiar Figures Test, a behavior rating scale. 
The OBG monograph (Bender, 1938) was also used for patients within psychiatric hospitals to differentiate between the functionally mentally ill and malingerers. However, later research by Pascal and Suttell (1952) found this function to be invalid.

Subsequently, Mehlman and Vatovec (1956), Bowland and Deabler (1956), and Stewart (1957) found that the Bender was reliable in differentiating between psychiatric and nonpsychiatric patients being admitted to the hospital. Regardless of differing findings, professionals continue to find the Bender to be of value in their evaluations and even prior to revision it remained a favored test in use (Piotrowski, 1995; Piotrowski \& Keller, 1989). Bender's scoring system evaluated the overall quality of each design on a scale that ranged from 1 to 5 on one design and from 1 to 7 on the other eight designs. Her scoring system is based on accuracy toward perfection of the design in a completion of a gestalt (Bender, 1938).

In an attempt to validate a direct measure of the emotional state of the individual or the projective use of the Bender, different psychologists developed specific scoring procedures The psychologists included Pascal and Suttell in 1952, Hutt and Briskin in 1960, Koppitz for children in 1963, Keogh and Smith in 1961 and Canter in 1976. These specific scoring procedures have been joined by more recent scoring systems such as the Advanced Psychodiagnostic Interpretation Scale by Rosenberg and Raphael in 2000 (Canter, 1968; Keogh, 1965; Pascal \& Sutter, 1952; Piotrowski, 1995). These diverse scoring systems have been criticized for insufficient reliability. Hutt's popular scale for determining psychopathology, developed in 1977, was found in 1983 to have 
questionable reliability and validity (Rossini, 1983). Keogh and Smith's scoring system did not provide normative data (Brannigan \& Decker, 2006). The result of the questionable validity and the numerous scoring systems has led to a slight decrease in the use of the OBG cards among professionals (Archer et al., 1991; Wilson \& Reschly, 1996). However, this situation has further led those in favor of the OBG to explore its uses and revise the test with the goal of standardization as an empirically valid measure (Brannigan \& Decker, 2003, 2006; Brannigan, Decker, \& Madsen, 2004; Reynolds, 2007).

The revision of the OBG was in process for many years, first at the American Orthopsychiatric Association then later at Riverside Publishing, which held the copyrights, with the work of many advisors and more than 25 years of collaboration (Brannigan \& Decker, 2006). Tolor and Brannigan in 1980 stressed the need for research on the OBG, not only to assess personality dynamics and psychopathology, but also to diagnose organic pathology, and predict school learning problems (Brannigan \& Decker, 2006; Brannigan, Decker, \& Madsen, 2004). Four main components were of importance during the revision process: (a) keeping the original nine designs, but increasing the number of designs; (b) inclusion of a memory procedure; (c) comparison of both the deviation and quality based scoring systems; and (d) obtaining results from a nationally representative sample for validity (Brannigan \& Decker, 2003, 2006). The Bender Gestalt II (BGII) was released for publication in 2003 by the Riverside Publishing Company. 
Along with the release of the BGII, the Global Scoring System (BGSS) was released as a recommended standardized measure by Riverside Publishing Company.

Revisions were also begun on the original Koppitz scoring system. Pro-Ed Publishing obtained the rights to the Koppitz scoring system and retained Cecil Reynolds to revise its scoring version. It was released in 2007 as the Koppitz 2: the Koppitz Developmental Scoring System for the Bender- Gestalt Test II (Reynolds, 2007). The Bender Gestalt II Global Scoring System (BGSS) and the newly revised Koppitz 2 developmental scoring system are now used as the preferred empirically validated measures for visual motor integration. However, they have not been researched beyond this use and thus may not be a valid measure for personality assessment. It is worth noting that the Koppitz 2 (Reynolds, 2007) has included the Emotional Indicators as an additional measure to assess personality. But this component was not included in the original norming process.

Only an unpublished dissertation from 2004 used the Bender Visual Motor Gestalt Test II (BGII) with the scoring of the Koppitz 2 Emotional Indicators. Fidal in 2004 examined the BGII, using the Koppitz 2 Developmental Scoring System and the Emotional Indicators Scoring systems on adolescents who had incidents of abuse versus those who reported no such history. Fidal found that the independent $t$ tests could not significantly differentiate between the groups (Fidal, 2004). More research is needed to compare the two methods to determine whether to support or retire the use of the Emotional Indicators in order to end the controversy about the original Koppitz 
Emotional Indicators. A literature search for the use of the BGII as an assessment in evidenced-based practice led to no findings. Thus this current research may be the first to address the practical use of the BGII with the BGSS and the Koppitz 2 Scoring System in clinical decision making using likelihood ratios (Koppitz, 1968, 1971, 1975).

\section{Statement of the Problem}

This study attempts to address one of the main deficits that can occur in the diagnosis and treatment of children and adolescents with mental-health issues: that is the difficulty in finding a direct measure of the child's functioning and internal state without either asking overly obvious questions or relying on information from significant others. Thus, this study will combine the task of the practitioner with that of the clinical researcher to determine the practicality for the more frequent use of the BGII. The OBG has been widely used as a projective screening device, as previously defined. However, most of the research was limited to adult pathology. Little research was conducted in regard to childhood pathology and less was directed toward the adolescent population (Belter, McIntosh, Finch, Williams, \& Edwards, 1989; Rossini \& Kasper, 1987). In children, the OBG was used mainly as a test of visual motor development and the research at the time supported this conclusion (Decker, Allen, \& Choca, 2006; Koppitz, 1971). However, the original Bender test, now the BGII has been revised to include more drawing items that increases the baseline and ceiling of the test, added the recall procedure and developed an empirically normed scoring system called the Global Scoring System (BGSS) for standard use among clinicians (Brannigan \& Decker, 2003, 
2006). It would be of benefit to clinicians if the revisions and new scoring systems contributed to the psychologist's ability to accurately diagnose. Furthermore, it would be beneficial to compare the newly revised BGII forms with another highly valid behavioral report measure that identifies childhood and adolescent diagnostic areas of concern in an attempt to revive the original purpose of the Bender as a psychodiagnostic technique. It also might be useful to compare the BGII findings with the newer clinical assessments available to psychologists such as the semistructured clinical interview.

In this study the results of the BGSS and the Koppitz 2 scoring systems for the newly revised Bender Visual Motor Gestalt II (BGII) test were compared with two clinical screening measures of mental disorders: the Child Behavior Checklist, known as the CBCL (Achenbach, 2004), and the Kiddie Schedule for Affective Disorders and Schizophrenia-Present and Lifetime Edition, known by the acronym KSADS-PL+ (Findling et al., 2001; Youngstrom \& Duaz, 2005; Youngstrom, Findling, Danielson, \& Calabrese, 2001). The CBCL and KSADS-PL+ have been previously compared to each other in research (Wassenberg, Max, Koele, \& Firme, 2004; Youngstrom et al., 2001). The current research is exploratory in nature as it will attempt to find relationships between the revised Bender Gestalt II with the CBCL and KSADS-PL+ used in the diagnosis of childhood psychopathology. In order to assess the clinical utility of these results, the BGII using the BGSS and Koppitz 2 data were compared to results derived from the previously mentioned valid measures of pediatric diagnostic decision making, the KSADS-PL+ and the CBCL. In the current study, two updated scoring procedures for 
the BGII test were compared, the BGSS and the Koppitz 2, to see if either provided clinicians with a valid, cost-effective, projective-screening measure that would aid in diagnosis of mental-health issues in clinical practice with children. 


\section{Research Questions}

RQ1. Is the BG II, using the BGSS and Koppitz 2 scoring measures, an effective psychometric screening tool to use with a clinically referred population of children aged 5 to 18 when compared to a commonly used screening instrument such as the Child Behavior Checklist (Achenbach System of Empirical Based Assessment [ASEBA], 2006)?

RQ2. Are the scores derived from the BGSS and the Koppitz 2 scoring systems effective measures for the diagnosis of the presence or absence of pathology in subject children and adolescents when compared to the Washington University version of the Kiddie Schedule for Affective Disorders and Schizophrenia (WASH-U-KSADS-PL+), a research diagnostic instrument?

RQ3. Are there significant relationships among the results derived from the BGII, using the BGSS and Koppitz 2 scoring systems, with final pediatric DSM-IV-TR diagnoses of LEAD consensus results following the KSADS-PL+?

RQ4. Are there any significant relationships between the BGSS and Koppitz 2 scores (including the Koppitz 2 Emotional Indicators) derived from the BGII when it is used with clinically referred children from 5 to 18 years of age?

\section{Research Significance}

The significance of this study lies in the joining of the BGII, BGSS scoring system with the KSADS PL+, a semistructured diagnostic interview, and was termed

"groundbreaking” research according to Gary G. Brannigan (personal communication, 
11/30/2006). Brannigan is the co-author of the Bender Visual Motor Gestalt Test Second Edition (BGII). Comparisons of the BGII with several behavioral rating scales (Belter et al., 1987; McCormick \&Brannigan, 1984) and pathology groups (Field, Bolton, \& Dana, 1982; Rossini \& Kaspar, 1987; Shapiro, \& Simpson, 1995) yielded varying findings. Shapiro and Simpson (1995) found that primary psychiatric diagnosis was not related to Bender performance when using the earlier Koppitz scoring system. However, these studies were conducted with the OBG and are now considered outdated. This study will examine individual patient drawings and emotional factors to determine the diagnostic value of assessing childhood pathology in relation to the BGII cards and the two scoring methods as previously mentioned. From this study, it is hoped that a simple test will eventually emerge providing a bridge between an extensive and laborious clinical evaluation and a parental checklist of subjective problems, thus providing another source of valid psychometric data to be used in psychological batteries. Such a test may prove to be an effective, additional diagnostic instrument in standard intake protocols.

This study was open to all children 5 to 18 years of age who sought mental-health services at a large community mental-health center in Cleveland, Ohio. It was part of a larger study which included profiles of 825 children. The July 2006 population of Cleveland was approximately 444,313, with an estimated median household income of $\$ 24,105$ (2006). The percentage of residents living in poverty was $32.4 \%$ in $2005(20.4 \%$ for White non-Hispanic residents, 39.3\% for African American residents, and 38.5\% for Hispanic residents). The racial makeup of Cleveland in 2006 was 51\% African American, 
$38.8 \%$ White non- Hispanic, 7.3\% Hispanic, 0.9\% American Indian, and 3.6\% other races, with $2.2 \%$ of the population listed as two or more races. The Cleveland Municipal School District is the largest school district in Ohio (City Data.com, 2009).

\section{Definition of Terms}

Base rate. This is a quantitative representation of a particular event occurring in a population or setting. Base rates in mental-health settings are usually based upon demographic information of the clients, and can be influenced by referral sources, geographical locations, previous diagnoses given by different clinicians, and the market area of the clinician. Base rate is the starting point in diagnostic decision making when using Evidence Based Practice assessment (Youngstrom \& Duax, 2005).

The Bender Gestalt II Global Scoring System. The BGSS was specifically designed to assess visual motor integration across a lifespan and aid in discriminating various types of learning, psychological, and neurological problems using the revised BGII (Brannigan \& Decker, 2003). It has been chosen because it represents the first standardization of the revised drawings of the OBG and is preferred by the BGII publishers, Riverside Publishing Company. The BGSS allows two phases of administering the BGII, the copy phase and the recall (memory) phase with a total score developed for each phase (Brannigan \& Decker, 2003).

Bender Visual Motor Gestalt Test II. The BGII is the test based on the original nine Bender drawings (OBG) plus seven additional drawings developed by Brannigan and Decker for the revised edition. These researchers added four cards to the beginning 
of the protocol to be used for children 4 to 7 years of age resulting in a total of 13 cards. Three additional cards were added to the end of the original protocol for the subjects over eight years of age resulting in a total of 12 cards.

The Child Behavior Checklist. The CBCL, developed by Achenbach and Rescorla (2001), is a rating scale commonly used to assist in making a pediatric psychological diagnosis. It assesses a broad range of behavioral symptoms found in children with emotional difficulties (Achenbach, 1991). The CBCL was revised in 2001 when two different forms were created: the CBCL for ages 1 to 5 years (CBCL/1 -5) and the CBCL for ages 6 to 18 years (CBCL/6-18; ASEBA, 2006). The CBCL uses three different forms for reporting: parent, teacher, and self-report. Rater diagnosis is based on how often each item has occurred currently or within the past 6 months for specific questions using a forced 3-point scale response form. There are two open-ended items for the individual to report additional problems. However, the open-ended items are qualitative and are not included in standard scoring (ASEBA, 2006). The CBCL provides $\mathrm{T}$ scores and percentile scores for different behavioral areas. These areas are divided into three competency scales (Activities, Social, School), a total competency scale, eight syndrome scales, two broad problem scales, and a total problem scale. The eight syndromes are aggressive behavior, anxious/depressed, attention problems, rulebreaking behaviors, social problems, somatic complaints, attention-deficit hyperactivity problems, oppositional-defiant problems and conduct problems (ASEBA, 2006).The broad problem scales are comprised of internalizing and externalizing problem scales. 
High T scores are scores above 70 on the problem scales and are indicative of pathology in that particular scale. However, resultant high $\mathrm{T}$ scores on the competency scales are indicative of mental resiliency and internal strengths of the individual (ASEBA, 2006).

Diagnostic Likelihood Ratio (DLR). The ratio of two proportions: the sample of people with a particular test result among all those who have a specific condition divided by the sample of people with the same test result among all those without the condition (Hamza, 2008). A DLR is the ratio of the posttest odds of having a particular diagnosis to the pretest odds of having that diagnosis among the general population based on a specific assessment (CHOI, 1998). This is calculated by obtaining the sensitivity and specificity of a particular test. Sensitivity is the probability of obtaining a positive test result among those with a true diagnosis. Specificity is the probability of obtaining a negative test result among those individuals without the particular diagnosis (CHOI, 1998).

Evidence-based practice. An effective treatment approach that is used for specific disorders based on systematic empirical research. Most areas of medicine, psychology, and sociology encourage the promotion of treatments based on empirical evidence (Luebbe, Radcliffe, Callands, Green, \& Thorn, 2007; Youngstrom \& Duax, 2005). Evidence-based practice incorporates the best research evidence available, patient preference and clinical judgment in an effort to combine clinical practice and research advancements in psychology to advance the field (Luebbe et al., 2007). 
KSADS-PL+ with LEAD percentages. The Kiddie Schedule for Affective Disorders and Schizophrenia for School-Age Children (K-SADS) is a semistructured interview for the psychiatric assessment of children and adolescents aged 6 to 18. It was adapted from the adult version, Schedule for Affective Disorders and Schizophrenia (SADS) developed in 1978 by Endicott and Spitzer (Ghanizadeh, Mohammadi, \& Yazdanshenas, 2006). There are currently three accepted versions of the KSADS. The Kiddie Schedule for Affective Disorders and Schizophrenia- Present and Lifetime Edition (KSADS-PL+) was originally developed by Puig-Antich and is the version used in this study (Ghanizadeh et al., 2006). The KSADS-PL+ version is a further modification that includes additional items sensitive to symptoms of depression and mania and has been used to assess the presence of 32 DSM-IV-TR diagnoses. Scoring allows the researcher to question for the presence or absence of various symptoms within essential DSM-IV-TR categories with further questioning based on the clinical judgment of the interviewer (Ghanizadeh et al., 2006). The current version includes items from the Young Mania Rating Scale as well as the mood disorders module originated at Washington University, (WASH-U-KSADS-PL+; Geller et al., 2001; Youngstrom, 2005).

The Koppitz-2 Visual Motor Index. This instrument is a measure of overall visual-motor integration skill and is defined as the ability to relate visual stimuli to motor responses in an accurate and appropriate manner based on the developmental scoring system of Elizabeth Koppitz and adapted for the Bender Gestalt II (BGII; Reynolds, 2007). The Koppitz 2 raw score is based on yes/no questions specific to common errors. 
On the Koppitz 2, each drawing may have several questions regarding errors, such as on Design 7, the columns are all slanted left to right, $N o=0, Y e s=1$; the more errors produced by the individual, the lower the score. The Koppitz -2 Visual Motor Index was an age-corrected deviation-scaled score set $(M=100, S D=15)$. This error- based approach is different than the BGSS which focuses on drawing accuracy in copying the original stimulus card. The categorical descriptive ratings were identical to the BGSS as previously described: $80-89$ is below average, 90-109 is average, and 110-119 is high average (Reynolds, 2007). The time to complete the drawings has been standardized for age and results are interpreted according to the number of errors (Reynolds, 2007). The older version of the Koppitz Developmental Scoring System (1975) was the most commonly used procedure to score the OBG designs produced by children ages 5 to 12 (Shapiro \& Simpson, 1995).

The Koppitz 2 adds an additional score to the BGII, which is the Koppitz 2 Emotional Indicators (EI). The EI score is based on the OBG and original research regarding emotional pathology. Koppitz (1975) developed the EI test when used as a projective measure to identify children with emotional problems (Reynolds, 2007). Unlike the Koppitz 2 Visual Motor Scores, the Emotional Indicators are added based on errors drawn. The Koppitz 2 Visual Motor Index scores and Emotional Indicators scores have been included in this study as they are popular for use among clinicians treating children and adolescents. 
Longitudinal Expert Evaluation of all Available Data (LEAD; Spitzer, 1983).

The LEAD assessment involves a formal review of all the data by an expert or team of experts (Klein, Ouimette, Kelly, Ferro, \& Riso, 1994; Pilkonis, Heape, Ruddy, \& Serrao, 1991). LEAD was held post interview and was a final review of all presenting data under the direction of a licensed child psychologist. LEAD was held in person, by telephone, or video conferencing with all raters reviewing the results of the KASDS-PL+, along with the child's supplemental information such as the child's history, family history, and school behaviors. Final diagnoses were made by consensus based on a degree of certainty by the assigned raters.

Pediatric Diagnostic Categories. The categories use the $D S M-I V-T R$ as the basis for the diagnosis and categorization of mental disorders for both research and clinical practitioners. Affective Disorders include both the unipolar and bipolar types of affective disorders. Behavioral disorders include Attention Deficit Hyperactivity Disorder (ADHD) and Disruptive Behavior Disorders. The Residual Disorder category includes Anxiety Disorders which further includes Panic Disorders, Obsessive Compulsive Disorder, Posttraumatic Stress Disorder, Phobias, or any other diagnosed anxiety disorder within the $D S M-I V-T R$. The Residual Disorders category may also include those individuals not diagnosed with any disorders from those major categories but who were still seeking mental-health interventions. These may include those with Adjustment Disorders, Psychotic Disorders, Learning Disorders or those individuals that have symptoms that do not meet any $D S M-I V$-TR Axis I criteria (Youngstrom et al., 2001). 
Psychometric screening tool. This is an instrument developed to determine minimum criteria for inclusion in a specified group without the loss of the reliability or accuracy of the instrument (Hildreth, 1945). A screening instrument is often meant to be a brief assessment within a larger more detailed examination.

Receiver Operating Characteristic (ROC). ROC is a statistical method which graphically plots the sensitivity or true positive rate against the false positives rate of the occurrence of a specific condition. This is done to illustrate the accuracy of a particular diagnosis using a specific measure versus the probability of an inaccurate diagnosis using that same measure (Zweig \& Campbell, 1993). The ROC analysis and the ROC curve are useful in the selection of optimal tests or measures and the dismissal of nonuseful ones based on a statistically derived discrimination threshold. The ideal prediction measure results in a 1.0 diagnostic likelihood ratio, which represents $100 \%$ sensitivity and $100 \%$ specificity regarding a particular diagnosis. This outcome is highly unlikely, however, as there are usually a number of false negative and false positive outcomes in diagnostic testing (Zweig \& Campbell, 1993).

Semistructured interview. This is a diagnostic assessment technique with specific standardized questioning areas. These include suggested questions to cover specific DSM areas while using the clinical knowledge and judgment of the rater to decide on appropriate interventions for each individual client (Geller et al., 2001). 


\section{Chapter 2: Review of Selected Literature}

The psychological interview and the psychological assessment battery are two methods used by clinicians in making psychological and psychiatric diagnoses. Once a diagnosis has been formulated the results of the interview and assessment battery can be further used in developing an effective treatment plan. There are three basic methods for diagnosing individuals, the more traditional, less structured, open-ended interview, the structured interview and the semistructured interview. The more unstructured clinical interview has given way to more formalized interviewing techniques in order to increase accuracy and accountability in diagnosis and treatment. Each method has its advantages and disadvantages. The unstructured interview is qualitatively based, whereas the structured interview is quantitative and symptom based, while the semistructured interview attempts to balance diagnostic accuracy with clinical meaning by combining aspects of both the unstructured and structured approaches (Jellinek \& McDermott, 2004). Additionally, the intervention of managed health-care panels and insurance policy constraints has changed the level of accountability for psychologists with the requirement to evaluate and diagnose based on the DSM-IV-TR (Cashel, 2002; Jellinek \& McDermott, 2004).

Cashel (2002) found that the training of clinicians to conduct psychological assessments has changed very little over the years even with changes in psychological training objectives which now focus more on evidenced-based practices (Luebbe et al., 2007). Practicing clinicians working with children have, in recent years, increased their 
use of structured observations, behavior rating scales, and shorter formats of intelligence measures (Archer et al., 1991; Cashel, 2002). These abbreviated measures and screening instruments also serve as a form of cost containment for psychologists (Archer et al., 1991). Cashel reported that many of the clinicians surveyed reported significant limitations placed upon them by outside sources. He further believed that this ultimately restricted effective diagnostic decision making. Clinicians find themselves bound by increased pressure to be more outcome and diagnosis based and less focused on process in order to quickly arrive at a diagnostic label.

Contrary to this tendency is the reality that appropriate diagnosis and early intervention in mental illness are not only necessary but also have led to improved outcomes in children with different clinical diagnoses, such as autistic spectrum disorders, attention deficit hyperactivity disorder, depression, and bipolar disorder (American Psychiatric Association, 2000; Charman \& Baird, 2002; Lipovsky et al., 1989; Valderhaug, \& Ivansson, 2005). For example, Charman and Baird (2002) found that increased recognition of symptoms by primary health practitioners, more frequent use of screening instruments by professionals, and evidence that intervention improves outcomes, have all contributed to the earlier diagnosis of autism from a mean age of 12 years to four years. Kamphaus et al. (2000) found that the expansion of school-based psychological services has led to an increased awareness of childhood mental-health issues and earlier identification of those in need of instructional and developmental services. 
Psychologists continue to have an important role in striving to maintain a high standard of care while adhering to the goals of achieving diagnostic accuracy and dealing with the demands of an ever-changing profession. Therefore, the initial diagnostic assessment becomes a pivotal point in the treatment process (Krueger \& Finger, 2001). A thorough diagnostic assessment should include detailed information on development, family and social history, parental description of everyday behavior, activities of the child, direct assessment of the child's communicative, intellectual and adaptive functioning, as well as the child's self-perception (Charman \& Baird, 2002; Elbert, \& Holden, 1987; Sourander et al., 2005). How psychologists obtain that information in the clinical interview has been the prerogative of the clinician. Originally, a subjective clinical interview process was employed, but this utilized open-ended questions and projective assessment measures to evaluate and diagnose (Jellinek \& McDermott, 2004). Under certain conditions, clinical judgment came less into play as the use of behavioral assessment tools, both formal and informal, became available to assist in information gathering and assess client functioning (Aklin \& Turner, 2006; Dryden, 1986). However, it still remains largely an area of individual preference for each clinician to decide exactly how to gather data, evaluate, and report findings.

Evaluated data and findings are then summarized into a clinical picture of the individual using language common to professionals and treatment specialists regardless of their theoretical orientations (Aklin \& Turner, 2006; Dryden, 1986). The DSM-IV-TR (American Psychiatric Association, 2000) is the current clinical resource manual that 
guides diagnostic decision making, and that allows professionals to have a common basis or language in which to initiate treatment, coordinate care, and discuss prognostic concerns (Morgan, Olson, Krueger, Schellenberg, \& Jackson, 2000). The authors of the $D S M-I V-T R$ do not claim to adhere to any particular theoretical framework (American Psychiatric Association, 2000). The $D S M-I V-T R$ is a working medically based manual used not only by psychologists but also by many other health care practitioners, and remains important in this constantly changing field of science. Therefore, it has been under constant revision since its inception, attempting to alleviate weaknesses in previous editions and to facilitate diagnosis of individuals based on new research findings (Morgan et al., 2000).

Achieving individual diagnostic accuracy especially with children and adolescents has been challenging and is also well documented (Aklin \& Turner, 2006; Gerber, Appleton, Dykeman, Sampson, \& Toews, 1994; Smith, Muir, \& Blackwood, 2004). Although children and adolescents present with their own particular mental-health issues, until recently research and treatment have relied heavily on adult research and treatment guidelines for diagnosis and treatment of these specific groups. This situation has lead to the likelihood of misdiagnosis or under-diagnosis within the field (Aklin \& Turner, 2006; Gerber et al., 1994, Tolan \& Dodge, 2005). For example, misdiagnosis based on adult guidelines can result in children receiving medications that do not produce the desired clinical effects and which may also result in serious negative outcomes. Misdiagnosis has also been related to longer and more costly treatment regimens. These negative aspects 
have resulted in a general lack of trust in the profession by both clients and their families (Aklin \& Turner, 2006; Smith et al., 2004). In an effort to lessen the likelihood of misdiagnosis and eliminate the need for costly testing, psychologists have begun to look at the use of clinical base rates and evidence-based practice, as previously discussed, when diagnosing children and adolescents (Youngstrom \& Duax, 2005).

To assist in diagnostic decision making, psychologists have often relied on the use of lengthy multimethod assessments to insure a more global perspective on the individual (Archer et al., 1991; Dryden, 1986). Multimethod assessments within a psychological test battery involve several informants' reports of the child's behavior, as well as direct measures of the child's intelligence and abilities. For many reasons, including possible denial, mistrust of professionals, or limited insight, children may not be the best reporters of their own problems. Therefore, professionals have resorted to obtaining information regarding children from other sources, often in the form of checklists (Achenbach, 1991). It is understandable that reports of children and adolescent behaviors elicited at school may differ from behaviors seen within the home and vice versa. Therefore, information regarding the parent-child relationship and the teacher-child relationship is thought to add to the global picture in an assessment (Achenbach, 1991; Achenbach \& Rescorla, 2001). Along with reported views of the child, a multimethod assessment would include psycho-educational testing instruments, personality profiles, behavioral checklists and mental status examinations. Direct assessment of a child's skills or functioning is obtained through intellectual evaluation and cognitive assessment. Performance results 
are standardized and compared to the results of a reference group, normed either for age or grade. Some of the more common tests include the Wechsler Intelligence Scales and the Woodcock Johnson Scales (Archer et al., 1991). Psycho-educational testing alone does not specifically address mental-health and pathological concerns and tends to focus only on intellect and ability. Thus, personality or psychopathology is often mainly assessed in the form of behavioral checklists as the increase in the use of empirical methods has caused psychologists to be more cautious in the use of projective measures (Cashel, 2002; Elbert \& Holden, 1987; Kamphaus et al., 2000).

Predoctoral internship programs offering psychological training for child psychologists have found that generally teaching methods have remained relatively stable over the past two decades (Archer et al., 1991, Cashel, 2002). However, changes within general clinical practice have occurred. These include as noted, school- based assessment, growth of abbreviated IQ measures, and popularity of behavioral measures over projective measures (Cashel, 2002; Elbert \& Holden, 1987; Kamphaus et al., 2000).

Clinical researchers have also become aware of the need to develop a thorough and efficient intake protocol geared toward accurate diagnosis of childhood mental-health disorders (Youngstrom, 2001). As previously discussed, psychology has traditionally utilized both objective and subjective measures to aid the clinician in reaching accurate diagnoses. Since the release of the original Binet intelligence test in 1908, assessment measures have expanded to include not only tests for intelligence, but also for achievement, vocational aptitude, personality, neuropsychological, development, and 
individual behavioral. Currently clinicians also use many different subjective, objective, self-report, checklist, or observational techniques. The development of the empirically based $D S M-I V-T R$ classification system of diagnosis has resulted in more reliance by clinicians on formal data collection of symptoms, identification of problem behaviors, and determination of functioning (First et al., 2004).

Therefore, the initial interview and diagnostic formulation become pivotal points in the treatment process. The interview or assessment method used to obtain pertinent and valid information should include the following: development, family and social history, parental description of everyday behavior, activities of the child, direct assessment of the child's functioning, and the child's self-perception (Charman \& Baird, 2002). A continual quandary for many practitioners is the need to get as much information as possible within a limited time frame and to balance the rigorous task of information gathering with that of cost effectiveness.

One evidenced-based method developed to help obtain accurate diagnoses and reduce clinician diagnostic error allows psychologists to use the statistically based method of probabilities used by medical professionals. The Diagnostic Likelihood Ratio (DLR) method was adapted for use by psychologists and incorporated the inclusive symptom approach of the $D S M-I V$-TR with quantitative analyses (Youngstrom \& Duax, 2005). This evidence-based approach to assessment allows the clinician to weigh the use of various costly testing measures against the likelihood of supporting or negating possible diagnoses. Evidenced-based practice if used in conjunction with accurate base 
rates and likelihood ratios can reduce the previously discussed conflict between costly batteries of tests and limited time for diagnosis. However, there is a twofold problem in incorporating this approach to assessment. The practicing clinician needs to know his client base rate which is based on the likelihood of anyone in his market area having the specific diagnosis as well as the best assessment measures to evaluate for that specific disorder. More simply stated, the question is whether a particular assessment is going to add to the clinician's fund of knowledge about a specific diagnosis and will aid in reaching an accurate diagnosis in a timely and cost effective manner.

\section{Research Focus}

The purpose of this study was to compare the BGII drawings, using the BGSS and Koppitz 2 scoring methods, with the diagnostic accuracy of the WASH-U-KSADS-PL+ interview and the popular CBCL measure to determine the diagnostic utility of the BGII as a decision making tool. In the current study, the BGSS and the Koppitz 2 scoring system for the BGII test were evaluated in a research setting to assess their contribution as projective screening measures that would aid diagnosis and treatment planning in clinical practice with children. It has been the hope of this researcher to begin a process leading to an increase in the clinical utility of the BGII not only as a developmental visual motor performance test but also as an additional effective assessment tool in a psychological test battery. Since this is exploratory research, the BGII, BGSS and the Koppitz 2 scoring results have been used. It was predicted that the BGSS and the Koppitz 2 would do equally well in screening children for visual motor integration errors. It 
remains uncertain whether they can effectively reach a diagnosis when compared with the WASH-U-KSADS-PL+ and the CBCL in a clinical sample of children. Both scoring measures of the Bender Gestalt II Test, although similarly normed by current research and measurement techniques, may yield different results.

The WASH-U-KSADS-PL+ (Youngstrom et al., 2005) is currently being used in an ongoing study "Assessing Bipolar Disorder: A Community Blend (ABACB)" to assess accuracy of childhood diagnosis with regard to childhood bipolar disorder in Cleveland, Ohio, through Case Western Reserve University. Permission to conduct the larger study was originally given by Case Western University Institutional Review Board in 2003 as a 5-year study comparing different instruments in diagnostic assessment for juvenile bipolar disorder. Addenda to this study have been routinely added during the yearly review process to compare various measures, both research and clinically driven. The University of North Carolina Institutional Review Board further reviewed this study in 2006 for data collection at an additional site. Once the IRB addendum was approved through Case Western Reserve University, this study was presented to the West Virginia University Institutional Review Board (Youngstrom, 2005). West Virginia University determined the data collection was archival and the present study was given exempt status. All Institutional Review Board releases can be viewed in Appendix A.

The major purpose of the Case Western Reserve University study with sites at Applewood Centers Inc., Case Western University, and University of North Carolina was to clarify the characteristic features of childhood bipolar disorder in children, to cross 
validate a childhood bipolar screening protocol for use in clinical settings, and investigate the developmental changes in symptoms across the age span using both cross sectional and longitudinal approaches (Youngstrom, 2005). Because one of the main purposes of the research study was the validation of potential screening measures, these screening tools were segregated from the LEAD assessment and reviewed and analyzed separately (Youngstrom et al., 2005). The BGII was one screening tool that was added in the fifth year of the study and was also segregated from the LEAD process (see Appendix B). If any of the Bender results had been found to be an adequate predictor of any specific diagnostic criteria then further analyses would have been conducted to determine its future use by developing a diagnostic likelihood ratio. In this study, a DLR was only performed for children with the diagnosis of ADHD based on the KSADS-PL + with LEAD results (Frazier, 2006; Jaeschke, Guyatt, \& Sackett, 1994; Youngstrom, 2006).

\section{The Bender Visual Motor Gestalt Test}

The OBG has been commonly used as a quick, simple, direct measure of the individual's internal psychological state (Archer et. al., 1991; Bender, 1938; Piotrowski \& Keller, 1989). The originally titled OBG, or the Bender Visual Motor Gestalt Test (Bender, 1938), was composed of simple geometric drawings developed by Max Wertheimer (1880-1943) in his perceptual psychology experiments and later formalized by Bender (Archer et al., 1991; Bender, 1938; Koppitz, 1975).

According to Bender, developmental maturation of drawing ability is an ongoing process that follows sequential stages, incorporating gross to fine-motor skills, visual 
imagery, and perceptual awareness and a developmental drawing tendency from vertical to horizontal movements, and finally from two dimensional to three dimensional awareness. These stages were all considered part of the maturational process leading to the more intricate representations found within a mature representational drawing that become a complete gestalt or perceptual integration (Bender, 1938). Bender theorized that a deviation within this maturational process would obviously lead to a disintegration of the original representation or errors between the drawing and its stimulus. As previously noted, psychologists have found this concept helpful in identifying those individuals who have not yet developmentally matured, or are delayed in their visual motor perception, as well as those who were once psychologically mature yet for various possible reasons may have lost such integration (Bender, 1938; Brannigan \& Decker, 2003, 2006; Koppitz, 1975 ; Reynolds, 2007).

The relationship of visual-spatial skills and working memory in areas of intelligence when correlated with tasks of executive functioning has been more recently addressed by Miyake, Friedman, Rettinger, Shah, and Hegarty, (2001). These researchers found that people who are good at complex visual-spatial tasks also perform better on executive function tasks which are crucial in regulating and controlling behavior (Miyake et al., 2001). Shapiro and Simpson (1995) found the Koppitz scoring system in disturbed adolescents with emotional and behavioral disorders did not measure intelligence, but was able to interpret maturational development, which correlates to the use of the BGII. The researchers evaluated a clinical group of adolescents 12 to 17 years of age and 
determined that visual motor skills continue to develop beyond the age of 11 and the scoring results were not found to be related to initial diagnosis, gender, or intelligence level of the adolescent (Shapiro \& Simpson, 1995). Their work with the original Koppitz scoring system provided useful information with regard to adolescents for this research study.

As previously stated, the OBG was one of the most popular assessment measures for more than 60 years and it was consistently listed as one of the top used instruments by psychologists when queried over those years (Archer et al., 1991; Brannigan \& Decker, 2003; Piotrowski \& Keller, 1989). There have been many researchers who cited the OBG as the criterion measure in their research (Brannigan \& Decker, 2003; Reynolds, 2007; Hutt, 1985). Horn and O’Donnell (1984) studied the early identification of learning disabilities and found that the OBG was effective in identifying those children classified as learning disabled as well as those with low achievement. The OBG was found to be useful in separating those adolescents diagnosed with impulsivity from those without impulse difficulties (Oas, 1984). The Bender Gestalt-Recall technique was found to be a valid measure of short-term visual memory in children and adolescents and comparable to the Coding recall measure on the WISC-III (Imm, Kim, Belter, \& Finch, 1991). Since the OBG has been used in over 1,300 published articles and 60 years of research, its strengths and weaknesses are well documented (Brannigan \& Decker, 2003).

The use of the OBG became popular and widespread even with cautions on its use as a projective and neurological test. It continues to be a preferred measure possibly 
because of its simplicity and short administration time (Bigler \& Ehrfurth, 1981). The popularity of the Bender as a clinical tool has led to the development of a plethora of scoring measures and interpretive manuals over the years, although some exhibit highly questionable reliability and validity (Brannigan, \& Decker, 2003; Hutt, 1985; Perticone, 1998).

As a result the broad utility of the OBG has come into question over time. Many psychologists have cautioned against wide use of the OBG for diagnostic evaluations (Bigler \& Ehrfurth, 1981) because of low reliability with regard to its use as a projective test (Naglieri \& Pfeiffer, 1992). However, it still remains one of the top 10 instruments used by psychologists (Piotrowski \& Keller, 1989). Thus, the OBG has had two preferred uses in a childhood population: first, as a visual motor development instrument and then as a projective measure for identification of certain psychological conditions in both children and adults (Rossini \& Kaspar, 1987).

\section{Current Use of the Bender Visual Motor Gestalt-Second Edition}

The Bender Gestalt II (BGII; Brannigan \& Decker, 2003) is a modification of the OBG and maintains the importance of the quality of the drawing as its basis of scoring. However, the number of designs was increased from 9 to a total of 16 (Brannigan \& Decker, 2003). Four of the new designs are to be given to children younger than 8 years old and precede the original set of drawings. When giving the Bender Gestalt II drawings to anyone 8 years old or above, there are three additional drawings that follow the original administration (Brannigan \& Decker, 2003). 
Once developed and refined, the BGII was renormed using a national representative sample obtained through the 2000 US census as well as clinical samples of selected diagnoses (Brannigan \& Decker, 2003). Another change in the BGII is that it now includes perception and motor subtests to detect specific problems separate from integrative processes for those individuals who perform below expectation (Brannigan \& Decker, 2003). In order to measure visual-motor integration skills in children and adults from four to 85 years of age, the BGII is administrated in two stages. The first consists of a Copy and Recall phase, followed by two supplementary tests (the Motor Test and the Perception Test).

On the BGSS, scoring is performed by conversion of raw scores to Standard Scores ranging from 40 to $160(M=100, S D=15)$. Scores can also be converted to percentile ranks, $t$ scores, $z$ scores, and age equivalents. Individuals scoring $\geq 1.3$ standard deviations below the mean Visual Motor Index of 100 were considered to be "mildly impaired" and those scoring more than 2 standard deviations below the mean were considered "significantly impaired" (Brannigan \& Decker, 2003). Above average performance on the BGSS and Koppitz 2 presupposes normal levels of both visualperceptual skills and fine-motor coordination. Below-average performance may be due to problems in the domain, the integrative process, motivation, attention, or related concerns (Reynolds, 2007). A recent study examined the use of the BGII with children diagnosed with ADHD. Results indicated that those with ADHD tended to do more 
poorly than a normal child group. However, these differences disappeared when intellectual level was statistically controlled (Allen, 2005).

Despite the 2003 revision of the OBG, it is still regarded as controversial to many in the field, as will be discussed in greater detail in subsequent sections. However, as noted in the purpose of this research there are those who hope to rectify this situation and establish the BGII as a clinically useful tool.

\section{The Koppitz 2 and the Koppitz Developmental Scoring System}

The Koppitz 2 scoring system for the BGII is a revision of the OBG Koppitz developmental scoring system developed in 1963 by Koppitz, a child psychologist (Reynolds, 2007). Koppitz (1975) developed her own specific instructions for administration and scoring using a developmental approach with scores based on errors found within the design construction (Perticone, 1998). Scoring was based on total number of errors throughout the OBG. The original Koppitz was revised by Reynolds, a colleague and personal friend of Koppitz. In her early research, Koppitz had concluded that both developmental and emotional results had an overall diagnostic value and represented a possible indication of emotional disturbance within an individual (Perticone, 1998). Koppitz (1975) was guarded about generalizing individual indicators as personality characteristics. She saw them as more of a guide for further inquiry (Perticone, 1998). Following the introduction of the BGII, the need for revisions in the Koppitz Developmental scoring system became clear, resulting in Reynolds developing the Koppitz 2 Developmental Scoring System. 
Along with the developmental scoring, Koppitz included a scoring manual for Emotional Indicators, to differentiate children having emotional difficulties from those appearing well adjusted (Perticone, 1998; Reynolds, 2007). The Koppitz Emotional Indicators were developed using empirical methods based on psychodynamic theory to distinguish children with serious emotional problems from those without problems. Koppitz (1975) reported that emotional indicators were clinical symptoms or signs that should be individually evaluated by the rater. A single indicator by itself may not indicate serious pathology; however, a single indicator may show a manifestation or tendency toward some disturbance. Therefore, indicators could occur separately or in combination. The emotional indicators identified for scoring on the OBG were confused order, wavy lines, dashes for circles, increasing size, large size of drawing, or overly small size of the drawing, fine line, overworked or reinforced lines, second attempt of a drawing, and use of two or more sheets of paper to complete the test. However, these remain controversial due to their projective nature (Koppitz, 1975; Perticone, 1998; Reynolds, 2007). Additionally, Koppitz (1975) cautioned that the indicators were not comparable or correlated to the developmental test score.

The Koppitz 2 authors reviewed earlier research supporting the emotional indicators based on over 500 studies from Koppitz and others. They removed those that were demonstrated to be age related, and then included two indicators that were of considerable empirical significance based on previous research (Reynolds, 2007). The Koppitz 2 emotional indicators added drawing a box around one or more designs and 
spontaneous elaborations or changes in the overall gestalt of the design to the 10 emotional indicators reported by Koppitz in 1975. This resulted in a final total of 12 emotional indicators that when found in a profile were to be taken into consideration for concern by the clinician (Koppitz, 1975; Reynolds, 2007; Rossini \& Kaspar, 1987). The original research for the Koppitz Emotional Indicators reported that three or more errors were indicative of possible pathology (Koppitz, 1975). However, the current Koppitz 2 Emotional Indicators scoring is quantitative with more than four considered to be high risk or of concern for emotional difficulties (Reynolds, 2007).

Archer et al. (1991) researched psychological test usage among psychologists and found that the OBG rated third among all assessment measures for use with adolescents. According to this research, the OBG was primarily reported as diagnostically useful in learning disability assessments. This leads to a larger question: Exactly what resultant data have been derived from the clinical use of the OBG and now the BGII? Is it a viable screening method that can add information to the diagnostic assessment interview? Or should the BGII be replaced by more structured diagnostic techniques to arrive at the same necessary information? Finally, is the BGII of diagnostic benefit to include as an assessment measure in children and adolescents?

Kiddie Schedule for Affective Disorders and Schizophrenia for School-Age Children The Kiddie Schedule for Affective Disorders and Schizophrenia for Children (KSADS) is a semistructured interview. Methods of clinical interviewing have changed since 1938 when the Bender cards were first introduced as a screening instrument (Aklin 
$\&$ Turner, 2006). In an attempt to reduce the high error rate, structured, and semistructured interviews have been developed to replace the open-ended, more unstructured interview process (Aklin \& Turner, 2006). The structured interview process provides systematized ratings, outlining specific behaviors and symptoms that need to be addressed using a standardized format. These standardized formats, however, have limitations. The structured interviews have been criticized for their inflexibility and lack of depth in obtaining information (Aklin \& Turner, 2006). Semistructured interviews offer more latitude to the clinician for the diagnosis of patient symptoms. Although the semistructured interviews alone may be less reliable due to the individual style of the clinician, the qualitative and quantitative analysis found that the combination of the semistructured interview and the use of empirical screening measures, may yield the deepest understanding of the client's situation (Aklin \& Turner, 2006; Findling et al., 2001; Findling et al., 2005).

The K-SADS is a semistructured diagnostic interview for children and adolescents (6-18 years) designed to assess current and past episodes of psychopathology according to $D S M-I V-T R$ criteria. The K-SADS is administered by interviewing the parent(s) and the child, and finally developing summary ratings which include all sources of information (parent, child, school, chart, and other collateral sources).

The KSADS-PL+ and the CBCL have undergone considerable review and found to have acceptable validity and reliability (Achenbach System of Empirically Based Assessment [ABESA], 2006; Achenbach, 1991; Ambrosini, 2000; Ghanizadeh et al., 
2006). However, they are very different instruments (ABESA, 2006; Aklin \& Turner, 2006; Chambers et al., 1985; Findling et al., 2001; Findling et al., 2002). The KSADS is a research derived instrument with limited use in clinical practice due to the cost and administrative time demands (Chambers et al., 1985). The CBCL, on the other hand, is both time and cost efficient. It consists of a behavioral checklist of distressing behaviors to the responding individual. It is generally used in clinical practice as a screening instrument (ASEBA, 2006).

The K-SADS is a semistructured interview that was developed in the late 1970s by Drs. Puig-Antich and Chambers (Ambrosini, 2000). The original version of the KSADS has gone through several modifications. The K-SADS PL+ includes the present and lifetime edition of the KSADS along with the Youth Mania Rating Scale questions to cover all possible symptoms of bipolar disorder (Findling et al., 2005; Gracious, Youngstrom, Findling, \& Calabrese, 2002). The K-SADS PL+ is DSM III-R (1987) and DSM-IV (1994) compatible and includes interviews with both the parent and child. The administration of the KSADS-PL+ takes approximately 90 minutes for each interview and can take longer depending on the number of supplemental diagnostic areas that are deemed significant (Ambrosini, 2000; Findling et al., 2001; Findling et al., 2005). The KSADS, as well as the KSADS-PL+, allows for clinical judgment based on the research diagnostic criteria and the placement of a specific symptom within the diagnostic criterion (Ambrosini, 2000, Findling et al., 2001, Findling et al., 2005). 
The Wash-U-KSADS-PL+ interview is based on DSM-IV-TR diagnostic criteria, and as previously discussed in regard to the $D S M-I V-T R$, there can be considerable overlap across diagnoses and different clinician interpretation of a symptom. Using the KSADS, KSADS PL+, or the WASH-U-KSADS-PL+ versions, can all be an arduous process for the clinician, because training for test administration focuses on a clear understanding and interpretation of various symptoms, administering the test must adhere to a strict protocol, and learning to interpret the interviewee's responses are all difficult tasks (Findling et al., 2001, 2005; Youngstrom et al., 2002). WASH-U-KSADS PL+ training requires both a didactic and interview-observer component in order to teach the interview process and insure scoring reliability (Ambrosini, 2000; Geller et al., 2001). The K-SADS PL+ is administered by interviewing the parent and the child and finally developing summary ratings, which include all sources of information. At some current research sites, all the raters then meet for a consensus of the different summary ratings to determine clinical diagnoses; this is known as the longitudinal expert evaluation of all available data or LEAD (Spitzer, 1983).

The LEAD assessment involves a formal review of all the data by an expert or team of experts (Klein et al., 1994; Pilkonis et al., 1991). LEAD is implemented following interviews and includes a final review of all presenting data under the direction of a licensed child psychologist at one of the current sites (Youngstrom et al., 2005). LEAD may be implemented in person, by telephone, or video conferencing with various raters reviewing the results of the KASDS-PL+. The final diagnoses are determined 
based on percentage of certainty by trained raters (Youngstrom, 2005). Although raters are highly trained, which enhances scoring reliability, the interviews are very time consuming, so the KSADS PL+ with LEAD, is not likely to be used in most clinical practices. It is, however, used for research purposes and compared with commonly used assessment measures (Findling et al., 2002; Findling et al., 2005; Youngstrom et al., 2005). The WASH-U- KSADS PL+ with LEAD is a thorough and lengthy interview. It can be useful in achieving accurate diagnoses using the $D S M-I V$-TR criteria (Ghanizadeh et al., 2006). As previously stated, it is, however, impractical for the practicing clinician due to its labor intensive administration and low cost efficiency.

\section{The Child Behavior Checklist}

The Child Behavior Checklist (CBCL) by Achenbach and Rescorla (2001) is a commonly administered checklist given to both parent and child to disclose current symptomology (Achenbach \& Rescorla, 2001, ASEBA, 2006). It assesses 120 emotional, a behavioral, social and psychological problem frequently reported by parents; and is meant to be part of multi-informant instruments of empirically based assessments originally developed by Achenbach (1991) and later revised by Achenbach and Rescorla (2001). It assesses a broad range of behavioral symptoms found among children with emotional difficulties (Achenbach, 1991). The revised CBCL resulted in the development of two different formats: The CBCL for ages 1 to 5 years (CBCL/1 -5) and CBCL for ages 6 to 18 years (CBCL/6-18) (Achenbach \& Rescorla, 2001, ASEBA, 2006). The Achenbach behavioral checklists are multiinformant instruments and have three different 
formats which include: parent, teacher, and self report forms. The Achenbach Youth Self Report Form is a standardized forced response test designed for 11 to 18 year olds to report their own strengths and areas of difficulties. Rater answers are based on accuracy of each item currently or within the previous 6-month period (ASEBA, 2006). The CBCL provides $t$ scores and percentiles for three competency scales (Activities, Social, School), total competency in these areas, internalizing problems scale, externalizing problems scale, and total problems scale. There are also eight syndromes that can be differentiated on the CBCL: aggressive behavior, anxiety /depression, attention problems, rulebreaking behaviors, social problems, somatic complaints, attention deficit hyperactivity problems, oppositional defiant problems and conduct problems (ASEBA, 2006). The CBCL was intended to serve as one component of a multimethod empirically based assessment and record children's competencies and deficiencies as reported by their parents, parent surrogates, or teachers. The CBCL provides clinical information relating to strengths and competencies as well as problems within the individual (internalizing or externalizing problems).

The CBCL had been found to be useful in assessing child symptomology based on clinical levels of internalizing problems and/or externalizing problems, and distinguishing attention deficit disorder from other disorders (Ivanova et al., 2007, Lengua, Sadowski, Friedrich, \& Fisher, 2001). In research by Ivanova and others, the CBCL was found to useful be across cultures using the eight-syndrome structure across 30 diverse societies throughout the world (Ivanova et al., 2007). However the CBCL, 
similar to the $D S M-I V-T R$, has been criticized for its overlapping of diagnoses and a tendency to over-estimate the co-occurrence of diagnoses (Lengua et al., 2001).

One study found the CBCL to be the most commonly utilized behavioral checklist among clinical and school psychologists for use with children (Cashel, 2002). In recent years, behavioral checklists and rating scales have replaced personality and projective measures as the instrument of choice among psychologists (Kamphaus et al., 2000). The popularity of behavioral checklists has been found to be related to their time efficiency, straightforwardness and ease in quantifying the results (Kamphaus et al., 2000). However as with any testing method, there are limitations. The self-report measures may raise questions of honesty and defensiveness of the reporter (La Fiosca \& Loyd, 1986). Validity of self-reports has been found to improve when there are different sources of measurement. The reports often include parent, child, and teacher versions when screening (Achenbach \& Rescorla, 2001). This researcher supports the use of behavioral checklists to aid in diagnostic decision making and finds the CBCL is an adequate assessment tool to use for children within this current study. Although the Youth Self Report (YSR) by Achenbach (1991) would have been an ideal comparison assessment, because it is geared for children older than 11, it was not used as a comparison measure due to the younger aged children assessed.

As described in this section the OBG has been used for many years and previous research into the BGII lays the ground work for the current study examining the utility of the BGII and the previously discussed scoring methods. This current study also supports 
the assessment tools of the WASH-U-KSADS-PL+ and the CBCL as diagnostic decision making tools. How these instruments are used in the current study will be further addressed within the procedure section of the next chapter. 


\section{Chapter 3: Method}

This study is comparative research using archival data from a 1-year subset of a larger 5-year study that included over 600 participants. This parent study aimed at investigating the effectiveness of screening measures in diagnosing bipolar disorder in children and adolescents. For my study, a total of 115 children were initially evaluated and 75 completed all the procedures of the research protocol comprising the KSADS$\mathrm{PL}+$, the CBCL, and the Bender Visual Motor Gestalt Test II. The data for all subsequent analyses are derived from these 75 participants, as will be clarified below.

\section{Participants}

Inclusion criteria for this research as well as the larger general study were (a) youth between the ages of 5 years and 18 years, (b) written consent and assent from both caregiver and client, (c) both caregiver and youth presented for the assessment, and (d) both caregiver and youth were functional English speakers. Participants for the larger study were 620 caregivers and youth invited from the intakes of a community mentalhealth center in Cleveland, Ohio, following a consecutive case-series design. A consecutive case series design reports the outcomes of a group of individuals or clients with a similar condition treated in the same manner (McKeon, Medina, \& Hertel, 2006). The parent study enrolled participants between July 2003 and March 2008, while my research, which focuses on the BGII as a screening instrument, covered a period of time from May 2007 through March 2008.

As noted my study reports findings for the 75 youth, ages 5 to 18 , with complete

data for all measures. The most common reason for missing data was no Koppitz scoring 
for the youth $(n=26)$ followed by a missing CBCL $(n=7)$. There were seven other individuals whose protocols were missing a variety of data points. Of the 75 youth completing all elements of the study the average age was $11.92(S D=2.56)$, with a median of three diagnoses, 64\% male, $84 \%$ African American, 11\% Caucasian, and 5\% other. Participants enrolled in the larger study over the 5-year time period between ages 5 -18 were on average 12.00 years old $(S D=2.67)$, with a median of two diagnoses, $89 \%$ African American, 7\% Caucasian, and 4\% other. All subsequent data points are derived using an $n=75$. Independent sample $t$ test using a $t$ test for the equality of means indicate that youth in the current study were not significantly different in age or comorbidity than other youth in the larger study $t(74)=.817, p=.076$. Chi-square analyses indicated no significant difference between those who received the BGII and those who did not in ethnicity, $x^{2}(3)=2.86, p=.41$. Chi-square analysis indicated no significant difference between those who received the BGII and those who did not in gender, $x^{2}(1)=1.67, p=$ 20 .

\section{Measures}

The Bender Visual Motor Gestalt II- Global Scoring System. This measure was designed to assess visual motor integration across the lifespan and aid in differentiating learning problems as well as psychological and neurological problems (Brannigan \& Decker, 2003). The BGII consists of the original Bender drawings plus additional drawings added by Brannigan and Decker (2003) to expand testable age range and provide more discrimination for levels of visual motor perception. Compared to the OBG, the BGII includes new items $(\mathrm{k}=16)$ on the BGII compared to $(\mathrm{k}=9)$ on the OBG, 
a memory recall phase, national norms, clinical validity studies, time estimates, quantitative and qualitative scoring, test observation forms, and co-norming with the Stanford-Binet (Brannigan \& Decker,2003). Both the original and new figures were based upon laws of perception postulated by Wertheimer (1923). Figures appear individually on numbered cards. Administration of the BGII consists of two phases: copy and recall. The copy phase measures visual motor integration. During the copy phase, cards with a single figure are shown to the individual client one at a time. Participants then copy each figure onto a blank paper with a pencil. Immediately after the copy phase, the recall phase occurs. The recall phase measures short-term memory. The individual draws as many of the figures as he or she can remember on a new blank piece of paper immediately following the copy phase.

For the BGII, the Global Scoring System (BGSS) replaced the plethora of alternate scoring systems that had been used for the OBG (Brannigan \& Decker, 2003). The BGSS is a measure to assess the accuracy of the BGII drawings. Scoring is based on clinician comparison of examinee drawings to examples in the manual (Brannigan, Decker, \& Madsen, 2004). Each figure is scored on a 5-point Likert-type rating scale (0 to 4). A 0 means the drawn figure did not represent the stimulus at all. A score of 4 indicates the drawn figure matches the stimulus nearly perfectly. Drawings are rated from both the copy and recall phases of administration using the BGSS. The BGSS produces a sum raw score of all items administered. The raw scores are then converted to standard scores based on similarly aged individuals within the norming sample (Brannigan \& Decker, 2003). Standard scores range from 40 to $160(M=100, S D=15)($ Brannigan \& 
Decker, 2003). Classification ranges based on standard scores are represented by the following: 145 to160, extremely high or extremely advanced; 130 to144, very high or very advanced; 120 to 129 , high or advanced; 110 to 119, high average; 90 to 109, average; 80-89, low average; 70 to 79, borderline delayed; 55 to 69 , mildly delayed or low; and 40 to 54, moderately delayed or extremely low (Brannigan \& Decker, 2003).

Validity and reliability of the BGSS were originally discussed by Brannigan and Decker (2003) during the test revision process. Construct validity of the BGSS is assessed by comparison with the Beery-Buktenica Developmental Test of Visual Motor Integration, Fourth Edition (VMI-IV). The BGSS standard scores demonstrate strong, positive correlations with the VMI-IV on both copy $(r=.65)$ and recall $(r=.44)$ (Brannigan, Decker, \& Madsen, 2004). Additionally, the BGSS was compared to tests of achievement and cognitive ability. The BGSS has demonstrated modest correlations with the Woodcock -Johnson II Tests of Achievement (Woodcock, McGrew, \& Mather, 2001). The BGSS shows modest correlational scores ranging from .27 to .53 for the Copy Phase, and .25 to .49 in the Recall phase (Brannigan \& Decker, 2003). A study examining the relationship between the BGSS and the Stanford Binet Intelligence Scales, Fifth Edition (Roid, 2003) corrected correlations between the BGSS and the SB-5 IQ scores ranged from .50 to .54 for the Copy phase and .45 to .48 for the Recall phase. Interrater reliability of the BGSS was extremely high, yielding a Kappa $=0.90, p<0.05$. Another measure of reliability of the BGSS was found using the split-half method for internal consistency; Spearman-Brown prophecy formula was $>0.91$, at all age ranges indicating a consistent and stable measurement (Brannigan \& Decker, 2003). As reported in the 
manual, the BGSS demonstrates adequate validity and reliability for measuring visualmotor integration (Brannigan \& Decker, 2003).

In addition to the BGSS, other researchers have used the revised Koppitz 2 by Reynolds (2007) to score the figures of the BGII. For this research, Koppitz 2 scoring occurs after administration of the BGII and BGSS scoring. The Koppitz 2 Emotional Indicators score was incorporated and scored along with the Koppitz 2 Visual Motor Integration scoring. The Koppitz 2 is a scoring system used to derive assessment data from the Bender Gestalt II (2003). It provides an overall visual-motor integration score that shows the ability to relate visual stimuli to motor responses in an accurate and appropriate manner (Reynolds, 2007). The Koppitz 2 Visual Motor Index uses an age corrected deviation scaled score set $(M=100, S D=15)$. As previously mentioned, the categorical descriptive ratings are identical to those of the Bender Visual Motor Gestalt Test II- Global Scoring system results. Koppitz found significant differences between children with learning or behavioral difficulties and those who demonstrated average performance (Brannigan \& Decker, 2003). Koppitz recognized excessive time taken for test completion as a possible indicator of psychopathology. The time to normally complete the drawings has been examined in previous research and found to be between a mean of 9 minutes to 14 minutes depending on age for the copy phase with standard deviations of 4 to 7 minutes on both the BGSS and the Koppitz 2 (Brannigan \& Decker, 2003; Reynolds, 2007). Time is often a reported variable in the testing interpretation and has been associated with number of errors and willingness to perform the test accurately. 
Brannigan and Decker (2003) conducted studies during their research standardization of the BGSS to investigate the relationship between that system and the Koppitz 2. Correlations with that system and the BGSS were .80 for the copy phase and .51 for the recall phase. The lowest Cronbach's alpha of the Koppitz 2 as compared to the BGSS based on age ranges was found in the 5-year age group at 0.77 . In other aspects of the norming study of the BGSS, age, gender and race variables were considered. When divided into gender and racial groups, the Cronbach's coefficients on the BGSS were found to be approximately 0.90 for ages 8 and above (Brannigan \& Decker, 2003). These were calculated as internal measures of the BGSS during the norming process.

Test- retest method of reliability over time shows an average correlational coefficient, $r=0.77$ with a range of 0.73 to 0.85 in the normative sample. Interrater reliability was found to be Cohen's kappa $=0.91$ and 0.93 for the two different protocols of the Koppitz 2 (Reynolds, 2007). The correlation coefficients for the original Koppitz Scoring System and BGSS were 0.80 for the Copy phase and 0.51 for the Recall phase (Brannigan \& Decker, 2003). It appears that the scoring systems are stable and measuring similar constructs, but not to the point of redundancy.

Koppitz 2 scoring is based on specific aspects in the different designs that are considered indicators for the presence or absence of perceptual difficulties. Tests are scored on a point system based on examiner affirmative responses to the representation of a specific item. (For example, for Design 5, 1 point $=y e s$, the two items touch or nearly touch). There is a possibility of 45 correct in the Koppitz 2 raw score. Raw scores are then converted to standard scores and are the basis for the Visual Motor Integration score. 
The Koppitz 2 extracts a further score from the Bender drawings which is known as the Koppitz 2 Emotional Indicators (EI). This score is based on the use of the BGII as a projective indicator to identify children with severe emotional problems. This usage derived from research data collected by Koppitz and others over many years (Reynolds, 2007). Koppitz identified 12 emotional indicators that she believed were of considerable significance to those children with severe emotional problems and not a reflection of intelligence alone (Reynolds, 2007). Those 12 emotional indicators (EI) are (a) confused order, (b) wavy line, (c) dashes for circles, (d) progressive increase in drawing size, (e) large size of drawings, (f) small size of drawings, (g) fine lines, (h) overworked or reinforced lines, (i) second attempts at drawing a design, (j) expansion, (k) box around a design, and (1) spontaneous elaboration or additions to the design (Reynolds, 2007). In developing the Koppitz 2, Reynolds added the EI as a supplemental test based on the original Koppitz research and results (Koppitz , 1963, 1971, 1975).

The Kiddie Schedule for Affective Disorders and Schizophrenia for School-Age Children (K-SADS). This measure is a semistructured interview that was developed by Puig-Antich and Chambers (1978). The KSADS allows the examiner to systematically inquire about symptoms of psychopathology in children and adolescents. Kaufman and others modified the original KSADS so that it also inquires about present and past episodes of psychopathology, becoming the KSADS-PL (Kaufman, Birmaher, \& Brent, 2003). This version demonstrates adequate reliability and validity as a clinical diagnostic measure (Kaufman et al., 2003). The Washington University KSADS (WASH-UKSADS) version was further modified to include additional symptoms and associated 
features of depression and mania (Geller et al 2001). The WASH-U-KSADS-PL + combines the KSADS-PL and the WASH-U-KSADS. Additionally the WASH-UKSADS-PL+ includes the Child Depression Rating Scale (Geller et al., 2001) and the Young Mania Rating Scale (Gracious et al., 2002). The WASH-U-KSADS-PL+ was the modification used in the current study and considered the reference standard in childhood bipolar research. In the current study, interrater reliability was established by training all research assistants to attain a Kappa coefficient $\geq 0.85$ at the symptom level and Kappa $=$ 1.0 at the diagnosis level. To do so, new research assistants were initially required to score five administrations with a certified rater followed by five interview administrations leading and scoring the interview with participation of a certified rater. Passing was scored at an item level Kappa $>0.85$.

Final diagnoses were given via the Longitudinal Evaluation of all Available Data. (LEAD) process (Spitzer, 1983). The process involves a formal review of all data by an expert or team of experts. Raters were blind to the final diagnoses for study the research questionnaires and BGSS results, but were later integrated with family history, prior treatment history, prior testing history, and other clinical observations under the supervision of licensed clinical psychologists. LEAD was conducted in person, by telephone conferencing, or video conferencing with all raters reviewing the results of the WASH-U-KASDS-PL+, along with child history, family history, supplemental information, and other screening results. Final diagnoses were then determined based on consensus of certainty by the raters. 
The Child Behavior Checklists, (CBCL) and Youth Self Report Form (YSR). These measures by Achenbach and Rescorla (2001) are rating scales of common Axis I psychopathology as rated by caregivers and self-report. The CBCL and the YSR assess a broad range of behavioral symptoms found among children with emotional difficulties (Achenbach, 1991). The CBCL and YSR were developed to serve as components of a multiinformant empirically based assessment. The CBCL was revised in 2001 and resulted in two different forms, the CBCL for ages 1 to 5 (CBCL/1 -5), and the CBCL for ages 6 to 18 (CBCL/6-18; ASEBA, 2006). Caregivers and youth respond to each item using the past 6 months as the time frame. Responses consist of a 3-point Likert-type scale (0 to 2), with 0 indicating never true, 1 indicating sometimes true, and 2 indicating always true. Raw sum scores are transformed to T scores $(M=50, S D=10)($ Rescorla $\&$ Wagner, 2001). The CBCL and YSR yield eight syndrome scales and three general scales. The eight syndrome scales are aggressive behavior, anxiety/depression, attention problems, rule-breaking behaviors, social problems, somatic complaints, attention-deficit hyperactivity problems, oppositional-defiant problems and conduct problems (ASEBA, 2006). The three general scales are internalizing, externalizing, and total problems. High $\mathrm{T}$ scores on the problem scales are indicative of pathology. T scores higher than 70 are in the clinical range, $\mathrm{T}$ scores of 64 to 70 are in the borderline clinical range, while less than 64 are in the healthy range (Achenbach \& Rescorla, 2001). While the CBCL and YSR are widely used and considered valid measures of psychopathology, some critique their use for having limited response choices and the inability of the clinician to query responses, thus limiting the amount of clinical information gathered (Barker \& Pistrang, 2004). 


\section{Examiners}

The researchers were predominantly predoctoral interns participating as part of American Psychiatric Association predoctoral internship research requirements. Five research assistants were full-time employees who had undergone extensive training. All research assistants were under the direct supervision of the principal investigator and coinvestigators. The raters administered the protocols independently once deemed reliably trained. The reliability of raters was established over 10 passed trials with a veteran examiner until a 0.85 coefficient Kappa interrater reliability was obtained (Findling et al., 2001, 2002, 2005; Youngstrom et al., 2001; Youngstrom et al., 2005).

\section{Procedure}

All youth and caregivers presenting for a mental-health intake were invited to participate in a more detailed assessment during the clinical intake session for the general clinic. If youth and caregivers agreed to participate (62\% agreed; primary reason for not participating was duration of the study), they were then scheduled to meet with a research assistant ( $57 \%$ attended). Institutional review of this study was conducted under board approval from the University Hospitals of Cleveland and the Case Western Reserve University Institutional Review Board. The West Virginia University Institutional Review Board approval was sought; the study was granted exempt status because of the use of archival data (Appendix A). Both caregivers and youth provided written and verbal consent.

The WASH-U-KSADS-PL+ was conducted individually and separately with the youth and caregivers. The youth completed all other measures with a separate research 
assistant while the caregiver was being interviewed. The caregiver completed all other measures with another research assistant while the participant was being interviewed. The BGII drawings were scored according to the BGSS and Koppitz 2 by both the research assistant who administered the measure and by a research assistant blind to the interview administration and testing results. No significant difficulties were reported by research assistants during administration of the BGII. Youth completed the copy phase of the BGII in 8 minutes and 32 seconds on average $(S D=4.3)$. As previously reported, the normal time to complete the drawings in previous research has been found to be between a mean of 9 to 14 minutes depending on age for the copy phase with standard deviations of 4 to 7 minutes on both the BGSS and the Koppitz 2 for interpretation of results in relation to errors (Brannigan \& Decker, 2003; Reynolds, 2007).

\section{Data Analyses}

Data from the ABACB study (Youngstrom, 2006) were analyzed in SPSS v. 16.0. Data analyses were performed on the BGII GSS scores, BGII Koppitz 2 scores, the CBCL syndrome and general scales, the WASH-U-KSADS+PL and LEAD results. The CBCL syndrome and general scales were correlated using Pearson correlations with the BGSS scores and Koppitz 2 scores to examine whether similar constructs were being measured. The BGSS scores and the Koppitz 2 scores were compared to diagnoses using independent sample $t$ tests, ANOVAs, and Receiver Operating Characteristic (ROC). Diagnoses were categorized into four hierarchical groups: Bipolar Spectrum Disorders, Depressive Disorder, Behavior Disorders, and Other Diagnoses. For example, if a youth 
was diagnosed with Bipolar II and ADHD- Combined type, then he/she would be placed in the Bipolar Spectrum Disorders group (Youngstrom et al., 2001).

Significant ANOVA results were further explored using post hoc tests; Tukey's HSD was used if variances among groups could be considered equal. Games-Howell was used if variances among groups could not be considered equal. Holm's step-down correction procedure was used to compare independent $t$ tests with hierarchical diagnosis groups.

The evaluator could then assess the BGSS and Koppitz 2 level of specificity and sensitivity with regard to specific measurement results as well as common childhood diagnoses by testing different cut-off scores related to those significant ANOVA findings. If any of the hypothesized results had been found, they could have demonstrated the utility of the Bender in diagnosis in evidence-based practice (Youngstrom \& Duax, 2005). The standard of using significant ANOVA findings in evidenced-based practice had already resulted in the development of Receiver Operating Characteristic (ROC) analyses to examine the diagnostic sensitivity and specificity of the assessment measures (Altman \& Bland, 1994b; Frazier, 2006; Jaeschke et al., 1994; Youngstrom, 2006).

The ROC analysis plots sensitivity and false alarm rate (1-specificity) to aid in the interpretation of scores and can separate cases from those identified as noncases. Sensitivity is the proportion of individuals identified by the test as positive for the diagnosis that had been previously positively classified for that diagnosis (Altman \& Bland, 1994a; Choi, 1998). A false alarm is the belief that something is positive but it is not; therefore an incorrect diagnosis may be assumed when the child does not actually 
have the disorder. Specificity is the proportion of individuals identified correctly without the diagnosis (Altman \& Bland, 1994a; Choi, 1998). An ROC plots the sensitivity/false alarm rate for each score thus producing a curve. A straight line indicates a 50/50 chance of being given the specific diagnosis and is considered random. Tests that do not discriminate above the level of random chance are of no use to clinicians (Zweig \& Campbell, 1993). ROC curves examined diagnostic efficiency by comparing the sensitivity and false alarm rate (1 - specificity) for each score (Altman \& Bland, 1994a). ROC results are determined by nonparametric methods that result in a decimal fraction that represents the area under the curve (AUROC). An AUROC of .50 would indicate chance performance.

In evidence-based practice, clinical decision making uses the diagnostic likelihood ratios derived from ROC analysis to improve the likelihood of a clinically significant diagnosis. Mathematically, this is a result of the Bayes' Theorem. Visually a nomogram can be used to simplify the process in clinical practice (Frazier, 2006; Jaeschke et al., 1994; Youngstrom, 2006). The use of diagnostic likelihood ratios, ROC, and nomograms are relatively new to the field of psychology yet have been used in determining the utility of medical procedures and tests for years (Frazier, 2006). Diagnostic likelihood ratios were calculated to aid in understanding the clinical significance. Using Bayes' Theorem, the diagnostic likelihood ratio is multiplied by pretest odds (or base rate) to determine the posttest odds. For ease of clinical use, the odds are presented as probabilities in percent form. If one uses a binary cut point (e.g., 
clustering all clients below a score and all others above a score), then the positive diagnostic likelihood ratio is sensitivity divided by 1- specificity for the cut point score.

In the current research and prior to statistical analysis, a power analysis was conducted using G Power (Erdfelder, Lang, \& Buchner, 1996). Power of 80\% was calculated a priori for the correlational results. A moderate correctional effect size was used $(r=.30)$. Results indicated that a sample of 67 clients was required for statistical utility. However, power of $80 \%$ was calculated a priori for the difference between group results using independent sample $t$ tests with a moderate effect size (Cohen's $d=0.5$ ). Results indicated a sample of more than 67 clients was sufficient and therefore an $\mathrm{N}$ of 75 or larger was sufficient.

This chapter has detailed the methods and procedures used in this study along with the rationale for these methods. As stated the data were gathered for approximately one-year in the duration of a larger 5-year study. The study was designed to fit into the framework of the larger study and the data collected was archival. 


\section{Chapter 4: Results}

The present study was conducted to examine the clinical utility of the Bender Gestalt Visual Motor Test II (BGII) as a diagnostic discriminator for young children and adolescents. Two different methods for scoring the BGII, the Global Scoring System (BGSS) and the Koppitz 2 Scoring System, were compared to determine whether the BGII is an adequate measure for identifying pathology in children at initial intake. Participants in the present study were administered the BGII during an interview process to assess clinical diagnostic accuracy among a variety of screening instruments. All data were converted to $\mathrm{T}$ scores with a mean of 50 and standard deviation of 10 for analysis.

Data from the current study were analyzed in SPSS v. 16.0. Data analyses were performed on the BGII GSS scores, BGII Koppitz 2 scores, the CBCL syndrome and general scales, the WASH-U-KSADS+PL and LEAD results. The CBCL syndrome and general scales were compared with the BGSS scores and Koppitz 2 scores using Pearson correlations to determine whether similar constructs were being measured. The BGSS scores and the Koppitz 2 scores were then compared to diagnoses using independent samples $t$ tests, to determine whether similar constructs were being measured. The BGSS scores and the Koppitz 2 scores were then compared to diagnoses using independent samples $t$ tests, ANOVAs, and Receiver Operating Characteristic (ROC).

\section{Research Question 1}

When compared to a common screening instrument such as the CBCL, the BGII using the BGSS and Koppitz 2 scoring methods will be useful in the diagnostic screening 
of a clinically referred population of children. Results of the BGSS scores are given first followed by results of the Koppitz 2 scoring. The BGSS scoring of the BGII was compared to the CBCL using Pearson correlations. The BGSS Copy T scores were not found to be significantly related to the CBCL Internalizing, Externalizing, and Total Problems T scores. The Bender GSS Recall T scores, $\left(r=-0.26, p<0.01, r^{2}=.07\right)$ have a negative relationship to the CBCL Total Problems T scores. Therefore, an increase in the total number of behavior problems was associated with a decrease in the recall scores. The BGSS Recall T scores demonstrated a significantly negative relationship to the CBCL Externalizing T scores $\left(r=-0.23, p=0.01, r^{2}=0.05\right)$. This indicates that as scores on externalizing or acting-out behaviors increased child recall scores decreased. The CBCL Internalizing T scores were found not to be significantly related to the BGSS Copy T scores $(r=0.002, p=0.98)$ or to the BGSS Recall T scores $(r=-0.08, p=0.36)$. The Internalizing and Externalizing CBCL scores were significantly correlated to each other $r$ $\left.=0.44, p>0.01, r^{2}=0.19\right)$. Not surprisingly, both the Internalizing and Externalizing scores were highly correlated with the Total Problems T scores $(r=0.76, r=0.89, p>$ 0.01, respectively). Table 1 displays the complete correlation matrix for the CBCL scores and the BGII using the BGSS scoring system.

Results of the CBCL on the larger study indicate that the clinically referred groups of children were in the clinically borderline range for emotional problems: CBCL Total Problems $(M=68.74, S D=8.82, N=782)$, CBCL Externalizing Behaviors $(M=$ 69.63, $S D=9.72, N=782)$, and CBCL Internalizing Behaviors $(M=63.48, S D=10.34$, $N=782$, in the larger study). This is between one and two standard deviations above the 
mean on the CBCL based on the larger 5-year study results. This is indicative of high number of symptoms as reported by the parent or primary caregiver at the time of initial intake.

As the sample group, the BGII, Copy T scores, when adjusted for age were $(M=$ 52.10, $S D=10.85, n=75)$, and the Recall T scores were $(M=50.98, S D=11.83, n=75)$. These results are in the average range for the BGSS and are not considered clinically significant. The overall mean differences between the CBCL and BGII are indicative of a significant difference between the measures. The mean scores between the CBCL and BGSS scores were compared using a dependent sample $t$ test as they both provide T scores and are comparable one to the other. The results suggest that the BGSS indicates on average less visual motor problems in the sample than the CBCL indicates psychopathology in the sample. However, it is important to note that the BGII was completed by the child (client) and the CBCL was completed by parent or primary caretaker.

Pearson correlation coefficients were computed between the BGSS Copy and BGSS Recall T scores and the CBCL. The CBCL scores and results are presented in Table 1. The BGSS Copy T score has a moderately high positive relationship with the BGSS Recall T score, $(r=0.49, p<.01)$. In Table 1 , BGSS Recall scores were moderately negatively related to the CBCL Externalizing scores, $(r=-0.23, p<.01)$ and Total Problems scores, $(r=-0.26, p<.01)$. These results suggest a negative relationship between client's ability to recall drawings and number of externalizing behaviors reported by their caregivers as well as total number of symptoms endorsed. 
Overall the sample group and the time to complete the BGII show a moderate positive association with BGII GSS Copy T scores, $r=.34, p<.05$. Time to complete the BGII was not significantly related to any of the CBCL T Scores, all $p<.05$.

Table 1

Pearson Product-Moment Correlation Matrix Between the BGSS Copy and Recall Scores and the CBCL Main Scores

\begin{tabular}{|c|c|c|c|c|c|}
\hline & Copy & Recall & $\begin{array}{l}\text { CBCL } \\
\text { internalize }\end{array}$ & $\begin{array}{l}\text { CBCL } \\
\text { externalize }\end{array}$ & $\begin{array}{l}\text { CBCL } \\
\text { total problems }\end{array}$ \\
\hline BGSS copy & - & & & & \\
\hline BGSS recall & .49 & --- & & & \\
\hline $\begin{array}{l}\text { CBCL } \\
\text { internalizing }\end{array}$ & .01 & -.09 & & & \\
\hline $\begin{array}{l}\text { CBCL } \\
\text { externalizing }\end{array}$ & -.16 & $-.23 *$ & $.44 * *$ & & \\
\hline $\begin{array}{l}\text { CBCL Total } \\
\text { problems }\end{array}$ & -.16 & $-.26 * *$ & $.76 * *$ & $.86 * *$ & --- \\
\hline
\end{tabular}

Further analyses of the BGSS and CBCL were performed using the syndrome scales of the CBCL; Table 2 displays the complete correlation matrix for the CBCL syndrome scales and the BGSS scores. The CBCL syndrome categories were (a) aggressive behavior, (b) anxiety/depression, (c) attention problems, (d) rule-breaking behaviors, (e) social problems, (f) somatic complaints, (g) thought problems, and (h) withdrawn depressed. 
The CBCL attention problems scale was found to be negatively related to the BGSS copy scores, $(r=-0.14)$ and negatively related to BGSS recall scores $(r=-.24, p=$ $.01)$. The strength of the association of the relationship between CBCL attention problems and Bender Copy scores was $r^{2}=0.42$. The BGSS recall score and the CBCL thought problems syndrome category were positively related, $(r=-.26, p=.01)$; suggesting a relationship between the ability of the child to recall the drawings and reporting thinking difficulties or reality based behaviors.

The Bender Recall scores were found to correlate to several of the subcategories of the CBCL. The Bender Recall scores were found to have a weak negative correlation with the subcategories of anxiety-depression, social problems, thought problems, attention problems, and rule breaking, all $p<.05$. Again this suggests a closer connection between BGSS recall scores and symptom scores than the copy phase of the BGSS.

Further analyses of the BGSS method of scoring with the CBCL were conducted on the clinical diagnoses scales. Results revealed that BGSS copy scores were not found to be significant for affective disorder, anxiety disorder, ADHD or oppositional defiant disorder. Again however, the BGSS recall scores were found to be significant in those children who met the diagnoses of ADHD using the CBCL findings, $(r=.19, p=.05)$. This suggests that as BGSS scores increase so do ADHD subscale scores. In other words, poor performance on the recall is associated with higher ADHD scores. Findings of BGSS compared to the CBCL clinical diagnoses are found in Table 3. 
Table 2

Pearson Correlational Matrix of the BGSS Scores and the CBCL Syndrome Scores

\begin{tabular}{|c|c|c|c|c|c|}
\hline Subcategory & $\begin{array}{l}\text { GSS } \\
\text { copy }\end{array}$ & $\begin{array}{l}\text { GSS } \\
\text { recall }\end{array}$ & $\begin{array}{l}\text { CBCL } \\
\text { internalize }\end{array}$ & $\begin{array}{l}\text { CBCL } \\
\text { externalize }\end{array}$ & $\begin{array}{l}\text { CBCL } \\
\text { total } \\
\text { problems }\end{array}$ \\
\hline $\begin{array}{l}\text { Anxiety/ } \\
\text { depression }\end{array}$ & .07 & -.13 & $.85 * *$ & $.40 * *$ & $.68 * *$ \\
\hline $\begin{array}{l}\text { Attention } \\
\text { problems }\end{array}$ & -.14 & $-.24 * *$ & $.47 * *$ & $.55 * *$ & $.71 * *$ \\
\hline $\begin{array}{l}\text { Aggressive } \\
\text { behaviors }\end{array}$ & $-.20 *$ & -.18 & $.45 * *$ & .93 & $.82 * *$ \\
\hline Social problems & -.07 & -.17 & $.61 * *$ & $.55 * *$ & $.75 * *$ \\
\hline Rule breaking & .01 & -.15 & $.33 * *$ & $.86^{* *}$ & $.71 * *$ \\
\hline Thought problems & -.13 & $.26^{* *}$ & $-.60 * *$ & $.53 * *$ & $-.74 * *$ \\
\hline $\begin{array}{l}\text { Withdrawn } \\
\text { behaviors }\end{array}$ & .09 & .13 & $.77 * *$ & $.31 * *$ & $.56 * *$ \\
\hline $\begin{array}{l}\text { Somatic } \\
\text { concerns }\end{array}$ & .04 & -.10 & $.69 * *$ & $.26 * *$ & $.50 * *$ \\
\hline
\end{tabular}


Table 3

Pearson Correlational Matrix Comparing BGSS and CBCL Diagnostic Scores

\begin{tabular}{lllll}
\hline & $\begin{array}{l}\text { Affective } \\
\text { disorder }\end{array}$ & $\begin{array}{l}\text { Anxiety } \\
\text { disorder }\end{array}$ & ADHD & ODD \\
\hline $\begin{array}{l}\text { GSS copy } \\
\text { scores }\end{array}$ & -.09 & -.17 & -.18 & -.07 \\
GSS recall & -.16 & -.08 & $-.19 *$ & .11 \\
scores & & & &
\end{tabular}

To determine whether the Koppitz 2 scoring of the BGII was associated with psychopathology, Pearson correlational coefficients were computed between the Koppitz 2 and the CBCL results using their respective T scores to determine if there were any relationships. The initial analysis compared the Koppitz 2 total VMI and total EI scores with the CBCL scores for internalizing, externalizing and total problems (Table 4). These findings indicated a weak but significant relationship between CBCL Total Problem and Koppitz EI scores. No other significant relationships were found between the different Koppitz and CBCL testing measures. 
Table 4

Pearson Correlational Matrix Comparing CBCL and Koppitz 2 Scores

\begin{tabular}{llll}
\hline & $\begin{array}{l}\text { CBCL } \\
\text { internalizing }\end{array}$ & $\begin{array}{l}\text { CBCL } \\
\text { externalizing }\end{array}$ & $\begin{array}{l}\text { CBCL } \\
\text { total problems }\end{array}$ \\
\hline Koppitz EI & .15 & .06 & $.18^{*}$ \\
Koppitz VMI & -.08 & -.16 & -.19 \\
\hline$* p>.05$ & & &
\end{tabular}

Further analyses comparing the Koppitz 2 findings with the CBCL subcategories resulted in the following findings as shown in Table 5. First, the Koppitz EI scores were found to be related to the Koppitz Total Score $(r=0.48, p \leq .05)$ and the Koppitz VMI Scores $(r=.31, p \leq .05)$. Koppitz Total Scores were found to be correlated to the Koppitz VMI Scores $(r=0.88, p \leq .05)$.

Koppitz 2 Total Scores were also found to be correlated to social problems, $(r=-$ $.23, p \leq .05)$, and aggressive problems on the CBCL, $(r=-.24, p \leq .05)$, Koppitz 2 EI scores were correlated with social problems, $(r=0.28, p \leq .05)$, and attention problems, $(r=.28, p \leq .05)$. The Koppitz 2 scoring methods were not found to be significantly correlated with anxiety-depression, withdrawal, somatic complaints, thought problems, rule-breaking group, internalizers, externalizers, or total problems groups. This suggests a connection between the number of Koppitz 2 EI exhibited by the child and the number of social and attention problems described by the caregiver on the CBCL. Koppitz 2 Total Scores were strongly related $(r=0.88, p \leq .05)$ as previously suspected. 
Table 5

Pearson Correlation Matrix Comparing Koppitz 2 Total Score, Total Emotional

Indicators, and Visual Motor Index to the CBCL Syndrome Scales

\begin{tabular}{|c|c|c|c|c|c|c|c|c|c|c|c|c|c|}
\hline & 1 & 2 & 3 & 4 & 5 & 6 & 7 & 8 & 9 & 10 & 11 & 12 & 13 \\
\hline $\begin{array}{l}\text { 1.Koppitz } \\
\text { total score }\end{array}$ & - & & & & & & & & & & & & \\
\hline $\begin{array}{l}\text { 2.Emotional } \\
\text { indicators }\end{array}$ & $-.48 *$ & - & & & & & & & & & & & \\
\hline 3. VMI & $\begin{array}{c}.88 \\
*\end{array}$ & $-.31^{*}$ & - & & & & & & & & & & \\
\hline $\begin{array}{l}\text { 4.Anxious- } \\
\text { depressed }\end{array}$ & -.07 & .19 & .01 & - & & & & & & & & & \\
\hline $\begin{array}{l}5 . \\
\text { Withdrawn }\end{array}$ & .22 & -.10 & .19 & $.50^{*}$ & - & & & & & & & & \\
\hline $\begin{array}{l}\text { 6. Somatic } \\
\text { complaints }\end{array}$ & .11 & -.01 & .18 & $.43^{*}$ & $.41^{*}$ & - & & & & & & & \\
\hline $\begin{array}{l}\text { 7.Social } \\
\text { problems }\end{array}$ & $\begin{array}{l}- \\
.23 \\
*\end{array}$ & $.28^{*}$ & -.12 & $.60 *$ & .17 & $.35^{*}$ & - & & & & & & \\
\hline $\begin{array}{l}\text { 8. Thought } \\
\text { problems }\end{array}$ & -.10 & .08 & -.09 & $.55^{*}$ & .20 & $.49^{*}$ & $.61^{*}$ & - & & & & & \\
\hline $\begin{array}{l}9 . \\
\text { Attention } \\
\text { problems }\end{array}$ & -.22 & $.28^{*}$ & -.11 & $.47^{*}$ & .16 & $.31^{*}$ & $.67^{*}$ & $.62^{*}$ & - & & & & \\
\hline $\begin{array}{l}\text { 10.Rule } \\
\text { breaking }\end{array}$ & -.09 & .09 & -.09 & $.37 *$ & .21 & $.34^{*}$ & $.51^{*}$ & $.51^{*}$ & $.46^{*}$ & - & & & \\
\hline $\begin{array}{l}11 . \\
\text { Aggressive }\end{array}$ & $\begin{array}{c}- \\
.24 \\
*\end{array}$ & .19 & -.20 & $.53 *$ & .11 & $.24^{*}$ & $.75^{*}$ & $.63^{*}$ & $.65^{*}$ & $.69^{*}$ & - & & \\
\hline $\begin{array}{l}12 . \\
\text { Internalizing }\end{array}$ & .00 & .09 & .06 & $.83^{*}$ & $.75^{*}$ & $.69^{*}$ & $.51 *$ & $.51^{*}$ & $.40^{*}$ & $.38^{*}$ & $.42 *$ & - & \\
\hline $\begin{array}{l}\text { 13. External- } \\
\text { izing }\end{array}$ & -.21 & .15 & -.17 & $.51^{*}$ & .16 & $.27^{*}$ & $.69^{*}$ & $.64 *$ & $.63 *$ & $.84^{*}$ & $.95^{*}$ & $\begin{array}{l}.42 \\
*\end{array}$ & - \\
\hline $\begin{array}{l}\text { 14.Total } \\
\text { problems }\end{array}$ & -.22 & .22 & -.14 & $.73 *$ & $.37^{*}$ & $.51^{*}$ & $.79 *$ & $.78^{*}$ & $.74 *$ & $.73 *$ & $.85^{*}$ & $\begin{array}{l}.71 \\
*\end{array}$ & $.89 *$ \\
\hline
\end{tabular}




\section{Research Question 2}

When compared to the research instrument of the WASH-U-KSADS-PL+, scores derived from the BGII BGSS and the Koppitz- 2 scoring systems will be found to be valid predictors of pathological symptom severity among the children studied. Again the results section is ordered by the two systems used, BGSS first and Koppitz 2 second.

Results of the BGII BGSS were compared to the diagnostic categories of the KSADS-PL+ using Pearson Product-Moment correlations. The current study compared the different scoring results with specific pediatric diagnostic categories on the KSADS$\mathrm{PL}+$ to determine if the BGII could be useful in identification of pathology. Although when compared with the KSADS-PL+ diagnoses the BGSS results showed no significant correlations (Table 6), additional analyses found that within the WASH-U-KSADS-PL+ semiinterview, the ADHD diagnosis was positively correlated with the ODD diagnosis ( $r$ $\left.=.27, p=.01, r^{2}=.07\right)$.

The Koppitz 2 scoring results were found to be associated with the diagnostic findings on the KSADS-PL+. The KSADS-PL+ diagnoses were further compared to the Koppitz 2 Subtotals, VMI and EI using the Pearson Correlational Coefficient analyses as presented in Table 7. When comparing the Koppitz 2 VMI scoring results with that of the KSADS-PL+ diagnoses, there were no significant correlations found at the $p=.05$ level. However, when comparing the Koppitz 2 Emotional Indicators with different KSADS- 
$\mathrm{PL}+$ diagnoses, there was a positive correlation between ADHD diagnoses and the EI scores $(r=0.30, p<0.01)$ with a strength of association of $r^{2}=.08$. 
Table 6

Pearson Correlation Matrix Comparing the BGII, BGSS and Koppitz2 Scores and KSADS-PL+ Symptomology

\begin{tabular}{|c|c|c|c|c|c|c|c|c|}
\hline & 1 & 2 & 3 & 4 & 5 & 6 & 7 & 8 \\
\hline 1. Time to complete & - & & & & & & & \\
\hline 2. BGSS score & $.34 *$ & - & & & & & & \\
\hline 3. BGSS recall & .20 & $.53 *$ & - & & & & & \\
\hline 4. Koppitz2 total score & .16 & $.72 *$ & .50 & - & & & & \\
\hline 5. Emotional indicators & .04 & $-.23 *$ & $-.33 *$ & -.48 & - & & & \\
\hline 6. Koppitz2 VMI & $.25^{*}$ & $.82 *$ & $.51 *$ & $.88 *$ & $-.31 *$ & - & & \\
\hline 7. KSADS depression -. 10 & .02 & .14 & .14 & -.19 & .01 & - & & \\
\hline 8. KSADS mania & -.06 & -.10 & -.05 & .04 & -.18 & -.07 & $.50 *$ & - \\
\hline 9. KSADS ODD & -.15 & -.04 & -.21 & .04 & -.09 & .04 & -.03 & .32 \\
\hline
\end{tabular}

Table 7

Pearson Product-Moment Correlations of the Koppitz 2 Scores and KSADS-PL+ Diagnoses.

\begin{tabular}{lllll}
\hline & $\begin{array}{l}\text { Bipolar } / \\
\text { KSADS }\end{array}$ & $\begin{array}{l}\text { ADHD/ } \\
\text { KSADS }\end{array}$ & $\begin{array}{l}\text { ODD/ } \\
\text { KSADS }\end{array}$ & $\begin{array}{l}\text { CD disorder/ } \\
\text { KSADS }\end{array}$ \\
\hline $\begin{array}{l}\text { Koppitz VMI } \\
\text { T score }\end{array}$ & -.05 & -.12 & -.11 & .08 \\
$\begin{array}{l}\text { Koppitz EI } \\
\text { T score }\end{array}$ & & & & \\
& -.09 & $.30 * *$ & .03 & -.18 \\
& & & & \\
\hline$* p>.05,{ }^{* *} p>.01$ & & &
\end{tabular}




\section{Research Question 3}

There will be significant relationships among the scoring results of the Bender Visual Motor Gestalt II and Koppitz- 2 depending on diagnostic categories when related to final LEAD results. Children with bipolar disorders will differ from those with unipolar disorder, anxiety disorders, behavior disorders, and ADHD scores derived from the BGSS or the Koppitz 2 scoring systems.

Using the Holm's step-down correction procedure, independent samples $t$ tests were conducted to determine whether there was a significant difference on each of the separate scales. The scales are (a) BGSS copy scores, (b) BGSS recall scores, (c) Koppitz 2 total score, (d)Total emotional indicators and (e) Koppitz 2 VMI between KSADS-PL+ diagnoses categories. The use of Holm's step-down correction was preferred because it maintains alpha at close to .05 overall and protects statistical power more than the common Bonferroni Correction method. When using this approach, there are four comparisons being made on each scale based on the following categories. The categories are (a) bipolar disorder versus all others, (b) ADHD versus all others, (c) conduct disorder versus all others and (d) ODD versus all others.

The BGSS Copy scores $(t(73)=1.05, p=.29)$, BGSS Recall Scores $(t(73)=$ $0.07, p=.94)$, Koppitz 2 Total Score $(t(73)=0.32, p=.78)$, Koppitz 2 Emotional Indicators $(t(73)=0.14, p=.10)$, and Koppitz 2 VMI $(t(73)=1.09, p=.42)$ did not significantly differentiate between those diagnosed with bipolar $(n=9)$ and all other disorders $(n=66)$. ROC indicated the BGSS Copy score $($ AUROC $=.40)$, BGSS Recall 
score $($ AUROC $=.49)$, Koppitz 2 Total Score $($ AUROC $=.47)$, Koppitz 2 Emotional Indicators $(\mathrm{AUROC}=.36)$, and the Koppitz $2 \mathrm{VMI}(\mathrm{AUROC}=.41)$ did not predict bipolar disorder significantly better than chance, at $p>.05$. The BGSS Copy score $(t(73)$ $=1.66, p=.10)$, BGSS Recall Scores $(t(73)=1.69, p=.10)$, Koppitz 2 Total EI $(t(73)=$ $1.79, p=.07)$, and Koppitz 2 VMI $(t(73)=1.34, p=.19)$ did not significantly differentiate between ADHD $(n=47)$ and all other disorders $(n=28)$. On the Koppitz 2 Total Score children and adolescents diagnosed with ADHD $(M=19.47, S D=8.44)$ had a significantly lower scores than all others $(M=24.93, S D=9.49),(t(73)=2.56, p=$ $.01)$. An error score was created for the Koppitz 2 Total Score. An error score was an actual score subtracted from the total possible score. Receiver Operating Characteristic (ROC) analyses were also conducted to determine whether any aspects of the researched scoring systems could predict any specific diagnoses compared to those not having the diagnosis in the sample group. This score was created to aid in the interpretation of the ROC analysis because it moved the ROC curve to the conventional top left instead of the bottom right of the diagram. Figure 1 displays the ROC curve that demonstrates that the number of errors significantly predicted an ADHD diagnosis over all other diagnoses $(\mathrm{AUROC}=.67) 95 \% \mathrm{CI}=[.54, .80]$

Using diagnostic likelihood ratios, sample participants who made more than 23 errors have a positive diagnostic likelihood ratio (DLR) of 2:1. Expressed as a probability, an odds ratio of $2: 1$ is $66 \%$. This is calculated by the following equation: probability $=$ odds $/(1+o d d s)$; using the formula shown with the current data: $2 /(2+1)$ 
$=.66$. This indicates that in a sample in which $50 \%$ of the youth have a diagnosis of ADHD, a positive test result increases the probability of the child truly having an ADHD diagnosis to $66 \%$, and represents the increased likelihood of an accurate diagnostic finding when the prior odds are modified by incorporating the diagnostic likelihood ratio as indicated by Bayes' theorem to calculate posterior odds. Negative diagnostic likelihood ratios can also be calculated by taking (1-sensitivity) divided by the specificity for the cut point score. Clients making less than 23 errors have a negative diagnostic likelihood ratio of 0.7. Again using the Bayes' Theorem, prior or base rate odds (1.0) are multiplied by the DLR (0.7) which equal posterior odds of 0.7 , which convert mathematically to $41 \%$ using the formula above $.7 /(1+.7)=.41$. This indicates that in the same sample of which $50 \%$ of the youth could have an ADHD diagnosis, a test result of less than 23 decreases the probability of a child having the diagnosis of ADHD to $41 \%$. 


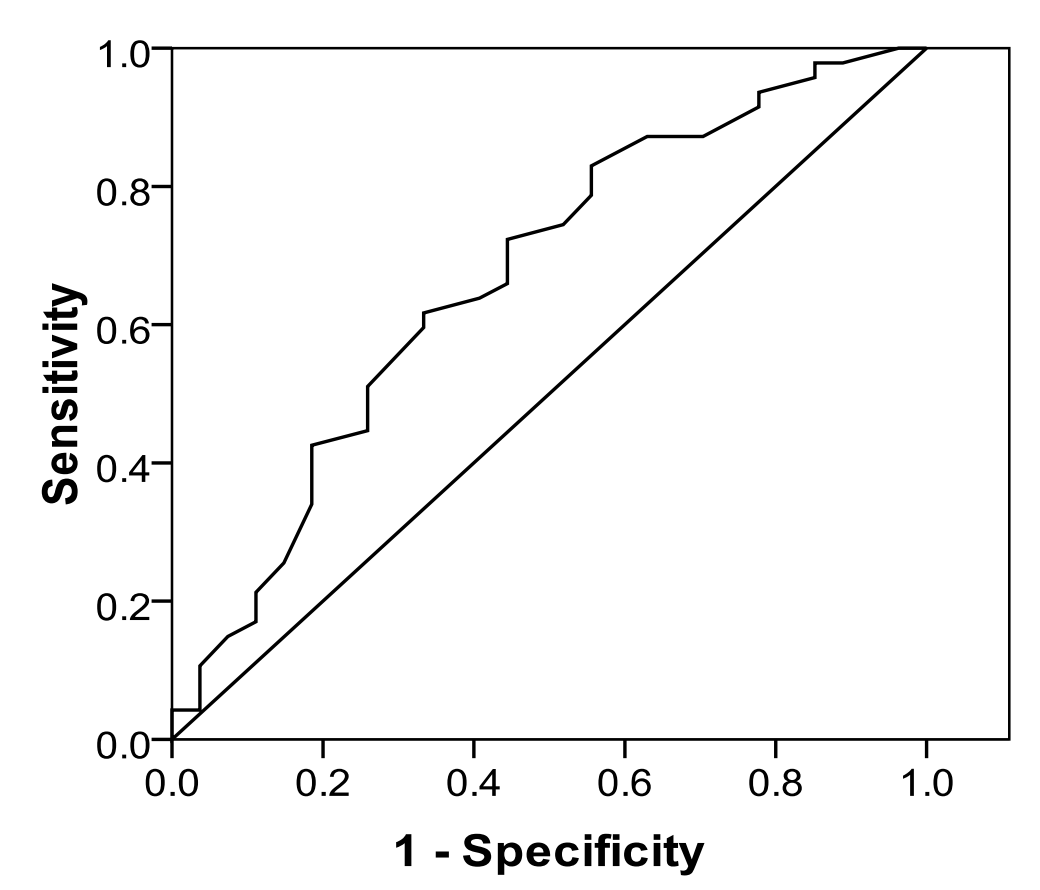

ADHD.

*Note: The solid diagonal line is chance (AUROC=.5).

Figure 1. ROC of errors made using the Koppitz 2 scoring system to predict.

The BGSS Copy score $(t(73)=.99, p=.99)$, BGSS Recall Score $(t(73)=.06, p=$ $.47)$, Koppitz 2 Total Score $(t(73)=-1.77, p=.08)$, Koppitz 2 Emotional Indicators $(t$ $(73)=1.82, p=.08)$ and Koppitz 2 VMI $(t(73)=-.62, p=.53)$ did not significantly differentiate between conduct disorder $(n=30)$ and all other disorders $(n=45)$. An ANOVA was performed using the mean of the BGSS Recall Scores across the KSADS$\mathrm{PL}+$ groupings of diagnoses found during LEAD. BGSS Recall Scores were found not to be significantly different with $\mathrm{F}(3,104)=0.76, p=0.97$. Due to a nonsignificant ANOVA and low shared variance among the different measures, additional analyses were not performed on this relationship. 
Using subscores from the Koppitz- 2 scoring systems of the Bender Visual Motor Gestalt Test II were compared with the final LEAD diagnoses groups to determine if any significant relationships exist. The ANOVA of the Koppitz -2 VMI -Scores were not significant. An ANOVA was performed with the Koppitz 2 Total EI scores and were found to be not significant. As previously shown in Table 5, the unipolar group had reported fewer emotional indicators than the disruptive behavior group and, though not significant, showed a difference between the cyclothymic/bipolar group and residual group. The average difference is only one emotional indicator. However, this difference of one emotional indicator is the difference between the normal range and the range of concern (Reynolds, 2006).

\section{Research Question 4}

Using hierarchical groups of diagnoses, four one-way ANOVAs indicated that the BGSS Copy Scores $(F(3,71)=0.56)$, BGSS Recall Scores $(F(3,71)=0.76)$, Koppitz 2 Total Score $(F(3,71)=0.46)$, Koppitz 2 Emotional Indicators $(F(3,71)=1.07)$ and Koppitz 2 VMI $(\mathrm{F}(3,71)=0.46)$ did not significantly differentiate sample participants with (a) bipolar, (b) unipolar depression, (c) behavior disorders, or (d) any other disorders, at $p>.05$. Due to nonsignificant ANOVAs and low shared variance among the different measures, additional analyses were not performed on this relationship to determine the level of specificity and sensitivity in relationship to clinical decision making when using the Bender Gestalt II together with the BGSS for ROC. 


\section{Chapter 5: Discussion}

In this study, the relationship between two scoring systems of the Bender Visual Motor Gestalt Test II and two empirically validated diagnostic instruments: the Child Behavior Checklist and the KSADS-PL+ were evaluated. The primary goal of this study was to determine the clinical utility of the Bender Gestalt II as a screening instrument in the initial intake process for identification of psychopathology in children and adolescents in a clinical setting. Specifically, is this nonverbal measure of visual motor integration useful as a projective indicator of individual psychopathology in children? Examination of the data indicates that aspects of both scoring systems for the Bender Gestalt II, the BGSS and the Koppitz 2 were found to have some limited utility in identifying psychopathology in children.

\section{Discussion of Hypotheses}

The initial hypothesis that the BGII, when compared with the CBCL, would be useful as a psychometric clinical screening instrument with children and adolescents was weakly validated by the current findings. These results indicate that when compared to the CBCL, the Bender Recall scores showed a significant negative correlation with the CBCL Externalizing scores. That is, children who exhibit a greater number or frequency of externalizing or acting out behaviors were found to have lower scores on the recall subtest. Bender Recall scores and CBCL Internalizing scores, reflective of such disorders as anxiety, depression and other dysfunctions that are not socially disruptive, 
lacked statistical correlation. This is expected considering previous studies have demonstrated that children who exhibit externalizing disruptive behaviors tend to have more difficulty with short-term memory than those with internalizing disorders (Kooistra, Crawford, Dewey, Cantell, \& Kaplan, 2005; Raggi \& Chronis, 2006). When comparing the CBCL with the BGSS and the Koppitz 2 results, several potentially useful findings did appear. Although the mean of the Bender results was within the normal range upon initial review, the individual CBCL mean was in the borderline clinical range. One possible reason for the difference between means is that the children completed the Bender Gestalt II themselves, whereas the caregiver was responsible for the CBCL results. In reviewing a series of reports, Youngstrom (2006) reported previously that parents are the most accurate reporters of symptoms. It is thus possible that the Bender is not assessing the same type of symptoms as children report differently than their caregivers. However, when subcategories of the test results were evaluated, a level of correlation began to emerge. Bender Recall T Scores were found to be associated with those of CBCL Externalizing and Total Problem scores. Results indicate that those with higher Externalizing scores and Total Problem scores found it more difficult to recall the presented Bender items. These recall items on the Bender Gestalt II are related to short-term memory and accuracy of drawing (Brannigan \& Decker, 2003). This is likely due to the relationship between attention and short-term memory, which is a difficulty commonly reported by caregivers and further supported by the Bender performance on recall tasks. 
On the CBCL syndrome scales, several further meaningful relationships can be identified. Bender Recall scores tended to be negatively related to those scoring high on ADHD symptoms, as well as those reporting with aggressive behavior problems. This was similar to the findings of Allen (2005) who found a relationship between youth with ADHD and low recall memory. Allen further found lower Bender Recall scores were positively associated with increased scores for rule-breaking behaviors. However, this was not supported in the current study when compared with the CBCL rule-breaking subcategory. In the current research, higher BGSS copy and recall scores were found to be negatively associated with CBCL attention scores. It is not surprising that those with difficulty paying attention would have a difficult time attending to accuracy of the drawings and later recalling the drawing to reproduce. Previous researchers found similar relationships with regard to the BGSS and diagnosis of ADHD and the specific symptom of impulsivity in children (Allen, 2005; Oas, 1984). Allen found that ADHD-diagnosed children performed more poorly overall on the Bender Gestalt II than normal children. Oas had previously found that results for adolescents with impulse disorders were significantly different from those designated as nonimpulsive on the Matching Familiar Figures Test, a behavior rating scale. It is important to report that the associated strength of most of these findings was considered weak but in the hypothesized direction. Since I did not have a nonclinical group as control and comparison, and this was a consecutive case series design, the current research findings are preliminary and not meant to be conclusive. 
A finding not previously reported but found in the current research is the strong correlation of reported increase in thought problems on the CBCL and high BGSS recall scores. The CBCL defines thought problems as obsessive thoughts, hallucinations, strange behaviors and atypical sleep patterns. One would think thought problems would impede the ability to maintain attend and recall. One possible reason for this finding may be that these children are highly aware of their surroundings or hyper vigilant, but are not outwardly expressing their thoughts and perceptions, thus resulting in their caregivers misinterpreting their behavior as odd or thought disordered.

A further hypothesis of this study postulated that when compared to a research instrument such as the KSADS-PL+, The Bender Gestalt II and the Koppitz 2 scoring methods results would be found to be valid measures of childhood pathological symptom severity. The KSADS-PL+ semistructured interview process represents both a qualitative and quantitative approach to data collection which affords clinicians a wider depth and scope of diagnoses. However, results from the Bender Gestalt II Global Scoring system were not found to be correlated with scores derived from the WASH-U-KSADS-PL+. There were no statistically significant findings on the BGSS when compared with the WASH-U-KSADS-PL+ for symptoms within the childhood diagnoses groups. As previously discussed, the KSADS-PL+ allows for more parental input than the forced choice standardized method of the CBCL and may have contributed to the lack of relationship between these two variables, as well as the varying results among these measures when further compared to the BGII scoring systems. Research comparing a 
testing measure such as the Child Behavior Checklist and the General Behavior Inventory with an interview method has been previously reported and the findings have been found useful in comparing a more subjective clinical impression to an empirically valid instrument (Youngstrom et al., 2000; Youngstrom et al., 2001, Youngstrom et al., 2005). As previously discussed, the Koppitz 2 scoring system resulted in several significant findings regarding individuals diagnosed with ADHD using the WASH-UKSADS-PL+ and the CBCL. As a screener for children with possible ADHD, the Koppitz 2 may aid in clinician decision making and thus be clinically useful. However, the Koppitz 2 Emotional Indicators (EI) were not found to be of benefit for diagnostic purposes in my research. As previously noted, the Emotional Indicators are those items that Koppitz recognized as highly indicative of pathology. Therefore, despite the weak results in my initial research, the Bender Gestalt II as a screener for ADHD diagnostic purposes in accompaniment with the WASH-U-KSADS-PL+ semistructured interview could be an avenue for future research.

A third hypothesis suggested that significant relationships would be found between the scoring systems of BGSS and the Koppitz 2 in relation to LEAD diagnostic categories. The final diagnostic categories were reviewed and determined during the formal LEAD process. These categories were defined previously as part of the bipolar study and therefore delineated mood disorders into two separate categories, Unipolar/ Depression and Cyclothymic/ Bipolar Spectrum Disorders. The Koppitz 2 and BGSS were unable to differentiate between the two mood disordered groups presented, although 
originally this was hypothesized. In previous research Shapiro and Simpson (1995) found that clients' primary psychiatric diagnoses were unrelated to their Bender performance. Yet when examining results obtained using the Koppitz 2 scoring method, they found Koppitz error rates to be weakly related to concentration. A more recent study by Allen (2005) indicated that ADHD-diagnosed children tended to do more poorly on the Bender Gestalt II than a normal group of children. However, these differences disappeared when a control variable for intellectual level was introduced. My current research findings also show that those diagnosed with ADHD have lower scores and thus could further support Allen's findings (Shapiro \& Simpson, 1995).

The final hypothesis of this study investigated expected differences between the BGSS and Koppitz 2 scores in a sample of 75 clinically referred children aged 5 to 18. These two scoring systems were found to be statistically significantly related to each other on most items, subcategories, and main scoring results with the exception of the BGSS Recall. Recall is more a measure of memory than drawing accuracy, and therefore the lack of statistically significant correlation is not surprising in comparison to the other measures within the scoring systems.

Research by Brannigan, Decker, and Madsen (2004) found a significant difference in the scoring approach of the Koppitz 2 versus the BGSS, leading them to describe the Koppitz 2 as "more lenient" in scoring than the BGSS. They noted that Koppitz 2 focused on specific aspects within a drawing whereas the BGSS scores the whole gestalt of the drawing. This finding was supported in the current research and may 
have resulted in some of the positive findings with regard to the Koppitz 2 and not the BGSS. However, it is not clear if the scoring systems' differences were the only factor in false positive findings or that other factors, such as a small sample size (75 out of 100 or more), may have contributed to the increase of false positives.

Additional research showed that when compared to the Beery-Buktenica Developmental Test of Visual Motor Integration- Fourth Edition (VMI-IV), the Koppitz 2 scoring system was less reliable than the BGSS. Nonetheless, the Koppitz 2 remains the preferred system for analysis of visual motor perception. In my research, I found that both systems when combined added the most clinical information in regard to visualmotor perception, as well as clinical understanding of the child's test taking behaviors during the administration of the BGII measure.

\section{Limitations of the Study}

The sample of this study initially consisted of a clinical group of 115 children. Several items from other assessments were found to be missing in the 115 individuals who completed the Bender Gestalt II test resulting in the lower final total sample of 75. Additionally, there was no control group of children without problems for comparison in this study. The majority of the subjects completing the protocol were 8 years and above, limiting the number of younger children for my research. This may have been a further limitation given the already small sample size. There may also have been an unwillingness of parents to answer certain items, such as those related to legal issues and the child's conduct on the day of the interview. 
There are the limitations related to the demographics of the sample. Participants in the present study were children living in urban Cleveland, Ohio. This may limit the possibility of generalization of findings to other geographic locations and populations. The sample was further limited by a lack of racial diversity. Participants were primarily African American $(n=63)$, consistent with previous referral patterns at the research facility (Youngstrom et al., 2000; Youngstrom et al., 2001; Youngstrom et al., 2005). Results may differ in larger metropolitan areas, more rural settings, or areas with a different demographic profile. For example, the data raised some questions about the impact of demographics, but the sample sizes were too small to come to any conclusions and further study may be warranted in this area. A further limitation is the failure to include a measure of intelligence within the study, which leaves the contributions of a potentially important variable unexplored.

Other limitations of this study are related to the clinical nature of the sample. The present sample consisted of children who were referred for treatment, and met the criteria for a $D S M-I V-T R$ diagnosis prior to interview. Upon agreement to be in the study, children and caregivers were both assessed according to the previously reported protocol. Data were not collected from anyone in the community who did not have a clinical relationship with the center. This suggests the possibility of a self-selection bias in addition to the restriction based on clinical status. Additionally, participants were grouped according to previously established clinical categories based on identifying children with bipolar spectrum disorders. Although a strict interview protocol was 
maintained, the fact that the larger study was geared toward the diagnosis of bipolar disorder in children makes it impossible to completely eliminate the possibility of examiner bias.

Another concern in the present study is that the scores on the CBCL were found to be two standard deviations above the normal average, suggesting that these children were exhibiting behaviors in the clinical range at the time of interview. Although this supports the previously stated limitation as a clinical group, it also should serve as a caution to the reader not to compare these findings to normal children and adolescents. In addressing the CBCL assessment for use in this study, previous research has found that the parent report is a good predictor of diagnostic concerns (Youngstrom et al., 2000). However, clinicians cannot always be certain about parental motivation or accuracy with regard to the identification of pathology. That is, a parent may exaggerate certain symptoms or frequency of behaviors while underreporting their perception of the child's internal thoughts and feelings. One more way to address this limitation may be to collect more self-report data from the child clients themselves. However, some studies suggest that child self-reporting also tends to be lacking in insight and underestimates problem behaviors (Youngstrom, 2006). Another avenue to address this limitation may be to have a second reporter complete the CBCL for each child.

\section{Implications for Practice}

The BGSS and the Koppitz 2, previously normed for developmental visual motor integration (Brannigan \& Decker, 2003, Reynolds, 2007), were found to be clinically 
useful when used with other assessments in diagnosing ADHD in children and adolescents. However, to be most useful, clinicians should consider the base rate of a possible ADHD diagnosis within their practices (Frazier, 2006). The use of the BGSS to evaluate more than visual motor difficulties could be of benefit to clinicians who have been trained in the use of the original Bender Gestalt (OBG) as a projective measure. Although this is initial research, the results of comparing the Bender Gestalt II with the CBCL and a semistructured interview, do suggest that the Bender has some clinical utility. The findings support the use of the Bender Gestalt II in children and adolescents as a screening instrument for visual perceptual difficulty, impulsivity, short-term memory recall, and organizational ability of the individual. The findings in the study do not support using the BGII as a purely projective measure. The BGII may be able to distinguish healthy children and adolescents from those with psychopathology as the OBG did, as previously reported by Bender (1938) and Koppitz (1975) along with other proponents of the Bender. However, that assertion cannot be made in this study because all children evaluated in this study were already from a clinical population.

Results of this study indicate that the Bender Gestalt II measures aspects other than simple visual motor perception and possibly begins to provide insight into the differentiation between types of psychopathology. Incremental validity improves when this test is combined with other standardized interview techniques. Therefore, if the clinician has difficulty in clearly making an ADHD diagnosis, the Bender Gestalt II may provide further clinical evidence. It was found that those with ADHD had several 
significant correlations with the CBCL and WASH-U-KSADS-PL+. Children diagnosed with ADHD tended to have poorer recall and lower overall Koppitz 2 scores than others. Although my study is exploratory in nature it hopefully represents the beginning of more empirically driven research into the utility of the BGII. This investigation, conducted in the framework of a clinical setting, is intended to have applied research implications. The clinical setting as a condition of the research may have created some further limitations. Evidence supporting the use of the Bender for clinical practice as a diagnostic instrument was not fully achieved within this study, as had been hypothesized. This study employed two scoring systems of the BGII: the BGSS published in 2003 and the Koppitz 2 published in 2007. Future researchers might wish to review other scoring methods that were developed to identify psychopathology using the older version of the Bender. Further research is needed to support the work of Brannigan (2003), who applied the previously-developed Hutt scales to the BGII and found it reliable and valid for personality assessment.

This study only reviewed the BGSS with the Koppitz 2 items for overall correlation but did not address scoring on individual items within those measures. The BGII test was selected for this study as a projective measure because it is time efficient and simple for clinicians to administer. It is not the only projective measure available to clinicians and it would be beneficial to explore other projective measures as well for possible increased utility as they relate to empirically standardized measures, such as the KSADS-PL+ and the CBCL. 
It would also be of benefit for follow up research to address incremental validity of the Bender Visual Motor Gestalt II in relation to other screening tests utilized in the larger ABACB study (Youngstrom, 2005), such as the General Behavior Inventory or the Wechsler Intelligence Scale for Children-IV. This would add to previous research by identifying strengths of the BGII test as a useful measure of client psychopathology. It also could further address the current hypothesis of the BGII as a useful projective measure in clinical diagnostic decision making. As the ABACB study has a longitudinal component with a 5-year follow-up, the Bender Gestalt II could be readministered and compared within an adult population (Garb, 2003). Therefore, further research could address situational changes and consistency among the drawings in an individual for this study over a 5-year time period. These findings could be very valuable in determining the long term usefulness of the BGII test as a clinical measure.

\section{Conclusions}

The field of psychology has evolved and grown significantly over the last 50 years and currently there are many more clinical assessment tools that are attractive to psychologists because of their perceived clinical usefulness. However, in some cases these are being pushed aside for measures and checklists that, while useful, may be too transparent in their questioning style. The publication of a revised Bender Gestalt II test in 2003 provided this researcher with the opportunity to look at the current clinical utility of one such historical diagnostic measure still in use. 
The use of the Bender Visual Motor Gestalt Test II as a direct measure of internal mental state was explored as a possible addition to the tools used in reaching a clinical diagnosis. It may provide an important "missing link" in the current evaluation process by helping to bridge the gap between parent and child symptom reporting. The current results suggest that the Bender Visual Motor Gestalt Test II is a less than adequate screening tool for diagnosing clients with unipolar depression versus bipolar disorders, but is somewhat more useful for identifying childhood disruptive behavioral disorders. With the publication of the Bender Gestalt II, the instrument has been standardized with specific instructions for administration and scoring. The test possesses adequate validity and reliability as a test of visual motor integration.

As discussed, findings regarding the use of the Bender II as a projective instrument were mixed depending on which scoring method was used. It appears that internalizing children versus those considered externalizers or those with high levels of total problems draw differently in overall quality, resulting in significantly different scores derived from the Bender Visual Motor Gestalt Test II Global Scoring System and Koppitz- 2 scoring systems. Thus it may be that the Bender drawings do assess some internal states of the individual and may reflect some individual Gestalt processes as originally proposed by Wertheimer and Bender (Bender, 1938). However results did not support the use of Koppitz Emotional Indicators as a measure of psychopathology.

The BGSS and Koppitz 2 results in regard to significant findings of the KSADS diagnoses were disappointing and limited. It was found that the only diagnosis 
consistently related to BGII performance was the diagnosis of ADHD. It was also found that Koppitz 2 scores based on error rates were more sensitive than the Bender Global Scoring system in clinician decision making regarding ADHD. The Global Scoring System identified Recall Scores to be related to ADHD, but was not found to be as predictive of that as the Koppitz 2.

In conclusion, the findings of the present study indicate that the Bender Gestalt II may have use beyond its traditional value as a measure of visual motor ability. Despite the mixed results in supporting the main hypotheses, the current findings are useful. This is an initial investigation that suggests that further research on the Bender Gestalt II as a screening tool for childhood pathology would be valuable. 


\section{References}

Achenbach, T. M. (1991). Manual for the Child Behavior Checklist/4-18 and 1991

Profile. Burlington: University of Vermont, Department of Psychiatry.

Achenbach System of Empirical Based Assessment. (2006). Achenbach Child Behavior Checklist. Retrieved from http:/ $\underline{\text { www.aseba.org/ }}$

Achenbach T. M., \& Rescorla, L. A. (2001). Manual for ASEBA School-Age Forms \& Profiles. Burlington, VT: University of Vermont, Research Center for Children, Youth, \& Families.

Aklin, W. M., \& Turner, S. M. (2006). Toward understanding ethnic and cultural factors in the interviewing process. Psychotherapy: Theory, Research, Practice, Training, 43(1), 50-64.

Allen, R. A. (2005). Utility of the Bender Gestalt -Second edition in the assessment of attention- deficit/hyperactivity disorder. Dissertation Abstracts International: Section B: The Sciences and Engineering, 65(11-B) 6035.

Altman, D, G., \& Bland, J. M. (1994a). Diagnostic tests 1: Sensitivity and specificity. British Medical Journal, 308, 1552-1561.

Altman, D. G., \& Bland, J. M. (1994b). Diagnostic tests 3: Receiver operating characteristic plots. British Medical Journal, 309, 188-194.

Ambrosini, P. J. (2000). Historical development and present status of the schedule for affective disorders and schizophrenia for school age children (K-SADS). Journal of the American Academy of Child and Adolescent Psychiatry, 39(1), 49-58. 
American Psychiatric Association. (2000). Diagnostic and Statistical Manual of Mental Disorders ( $4^{\text {th }}$ ed., text revision). Washington, DC: Author.

Archer, R. P., Maruish, M., Imhof, E. A., \& Piotrowski, C. (1991). Psychological test usage with adolescent clients: 1990 survey findings. Professional Psychology: Research and Practice, 22, 247-252.

Barker, C., \& Pistrang, N. (2004). Quality criteria under methodological pluralism: Implications for conducting and evaluating research. Retrieved from http://www.ucl.ac.uk/publications/papers.

Belter, R.W., McIntosh, J. A., Finch, A. J., \& Williams, L. D. (1989). The Bender Gestalt as a method of personality assessment with adolescents. Journal of Clinical Psychology, 45, 414-423.

Bender, L. (1938). A Visual Motor Gestalt Test and its clinical use: Research monographs No. 3. New York, NY: The American Orthopsychiatric Association. (Original work published 1938).

Bigler, E. D., \& Ehrfurth, J. W. (1981). The continued inappropriate singular use of the Bender visual motor gestalt test. Professional Psychology: Research and Practice, 12, 562-569.

Bowland, J. A., \& Deabler, H. L. (1956). A Bender-Gestalt diagnostic validity study. Journal of Clinical Psychology, 12 (1), 82-84.

Brannigan, G. G., \& Decker, S. L. (2003). The Bender Visual-Motor Gestalt Test (2nd ed.). Itasca, IL: Riverside. 
Brannigan, G. G., \& Decker, S. L. (2006). The Bender-Gestalt II. American Journal of Orthopsychiatry, 76 (1), 10-12.

Brannigan, G. G., Decker, S. L., \& Madsen, D. H. (2004). Innovative features of the Bender Gestalt II and expanded guidelines for the use of the global scoring system. Itasca, IL: Riverside.

Canter, A. U. (1968). BIP Bender test for the detection of organic brain disorder: Modified scoring method and replication. Journal of Consulting and Clinical Psychology, 32, 522-526.

Cashel, M. L. (2002). Child and adolescent psychological assessment: Current clinical practices and the impact of managed care. Professional Psychology: Research and Practice, 33, 446-453. doi:10.1037/0735-7028.33.5.44

Charman, T., \& Baird, G. (2002). Practitioner review: Diagnosis of autism spectrum disorder with 2-and 3-year old children. Journal of Child Psychology and Psychiatry, 43, 289-305.

Choi, B. C. K. (1998). Slopes of a receiver operating curve and likelihood ratios for a diagnostic test. American Journal of Epidemiology, 148, 1127-1132.

Decker, S. L., Allen, R., \& Choca, J. P. (2006). Construct validity of the Bender Gestalt II: Comparison with Wechsler Intelligence Scale for Children -III. Perceptual Motor Skills, 102(1), 133-141.

DeClercq, B., DeFruyt, F., Van Leewen, K., \& Mervielde, I. (2006). The structure of maladaptive personality traits in childhood: A step toward an integrative 
developmental perspective for DSM-V. Journal of Abnormal Psychology, 115, $639-657$.

Dryden, W. (1986) Eclectic Psychotherapies: A critique of leading approaches. In J.C. Norcross (Ed.) Handbook of Eclectic Psychotherapy (pp. 353-375). New York, NY: Brunner/Mazel.

Elbert, J. C., \& Holden, E. W. (1987) Child diagnostic assessment: Current trends in clinical psychology internships. Professional Psychology: Research and Practice, $18,587-596$.

Erdfelder, E., Faul, F., \& Buchner, A. (1996). GPOWER: A general power analysis program. Behavior Research Method, Instruments \& Computers, 28, 1-11.

Fidal, C. A. (2004). Examining abuse indicators on the Bender Gestalt Test. Dissertation Abstracts International: Section B: The Sciences and Engineering, 64 (8-B), 4003.

Field, K., Bolton, B., \& Dana, R. H. (1982). An evaluation of three bender-gestalt scoring systems as indicators of pathology. Journal of Clinical Psychology, 38, 838-842.

Findling R. L., Gracious, B. L., McNamara, N. K., Youngstrom, E. A., Demeter, A. C. A., Branicky, L. A., \& Calabrese, J. R. (2001). Rapid, continuous cycling and psychiatric co-morbidity in pediatric bipolar I disorder. Bipolar Disorders, 3, 202210. 
Findling, R. L., Youngstrom, E. A., Danielson, C. K., DelPorto-Bedoya, D., PapishDavid, R., \& Townsend, L. (2002). Clinical decision-making using the General Behavior Inventory in juvenile bipolarity. Bipolar Disorders, 4, 34-42.

Findling, R. L., Youngstrom, E. A., McNamara, N. K., Stansbrey, R. J., Demeter, C. A., Bedoya, D., ... Calabrese, J. R. (2005). Early symptoms of mania and the role of parental risk. Bipolar Disorders, 7, 623-634.

First, M. B., Pincus, H. A., Levine, J. B., Williams, J. B., Ustun, B., \& Peele, R. (2004). Clinical utility as a criterion for revising psychiatric diagnoses. American Journal of Psychiatry, 161, 946-954.

Frazier, T. (2006). Evidence based assessment of Attention Deficit Hyperactivity Disorder. Cleveland, OH: Applewood Centers.

Garb, H. N. (2003). Incremental validity and the assessment of psychopathology in adults. Psychological Assessment, 15, 508-520. doi:10.1037/1040-3590.15.4.508

Geller, B., Zimmerman, B., Williams, M., Bolhofner, K., Craney, J. L., Delbello, M. P., \& Soutullo, C. (2001). Reliability of the Washington University in St. Louis Kiddie Schedule for affective disorders and schizophrenia (WASH-U-KSADS) mania and rapid cycling sections. Journal of Academy of Child and Adolescent Psychiatry, 40, 450-455.

Gerber, S., Appleton, V., Dykeman, J.C., Sampson, D., \& Toews, J. (1994). The vital balance revisited or the resolution of the Counseling Profession's identity split. 
Journal of Counseling Psychology, 37, 2-14. (ERIC Document Reproduction Service No. ED373279)

Ghanizadeh, A., Mohammadi, M. R., \& Yazdanshenas, A. (2006) Psychometric properties of the kiddie schedule for affective disorders and schizophrenia-present and lifetime version. Biomedcentral Psychiatry, 6(10). Retrieved from http:// www.biomedcentral.com/1471-244X/6/10

Gracious, B. L. Youngstrom, E. A., Findling, R. L., \& Calabrese, J. R. (2002). Discriminative validity of the parent version of the young mania rating scale. Journal of the American Academy of Child and Adolescent Psychiatry, 41, 13501359.

Hamza, T. H. (2008). Meta analyses of diagnostic test evaluation data: Random effects approaches. Annals of Internal Medicine, 149, 889-897. doi: 978-90-9023002-3.

Horn, W.F., \& O’Donnell, J.P. (1984). Early identification of learning disabilities: A comparison of two methods. Journal of Education Psychology, 76, 1106-1110.

Hothersall, D. (1995). History of psychology (3rd ed.). New York, NY: McGraw-Hill. Hutt, M. L. (1985). The Hutt adaptation of the Bender-gestalt test (4th ed.). Orlando, FL: Harcourt Brace Jovanovich.

Imm, P.S., Kim, Y.F., Belter, R.W., \& Finch, A.W. (May 1991). Assessment of short term visual memory in child and adolescent psychiatric inpatients. Journal of Clinical Psychology, 47, 441-443. 
Ivanova, M. Y., Achenbach, T. M., Dumenci, L., Rescorla, L.A., Almqvist, F., Weintraub, S., ... Frigerio, A. (2007) Testing the eight syndrome structure of the Child Behavior Checklist in 30 societies. Journal of Clinical Child and Adolescent Psychology, 36, 405-417.

Jaeschke, R., Guyatt, G. H., \& Sackett, D. L. (1994). Users' guides to the medical literature: III. How to use an article about a diagnostic test: Section B: What are the results and would they help me in caring for my patients? Journal of the American Medical Association, 271, 389-391.

Jellinek, M.S., \& McDermott, J.F. (2004). Formulation: Putting the diagnosis into a therapeutic context and treatment plan. Journal of the American Academy of Child and Adolescent Psychiatry, 43, 913-917.

Kamphaus, R.W., Petoskey, M. D., \& Rowe, E. W. (2000). Current trends in psychological testing of children. Professional Psychology: Research and Practice, 31(2), 155-164. doi: 10.1037/0735-7028..31.2.155

Kaufman, J., Birmaher, B., \& Brent, D. (2003). Schedule for affective disorders and schizophrenia for school-age children- Present and lifetime version (K-SADSPL): initial reliability and validity data. Journal of American Academy of Child Adolescent Psychiatry, 36, 980-988.

Keogh, B. (1965). School achievement associated with successful performance on the bender gestalt test. Journal of School Psychology 3(3), 37-40. 
Klein, D. N., Ouimette, P. C., Kelly, H. S., Ferro, T., \& Riso, L. P. (1994). Test-retest reliability of team consensus best-estimate diagnoses of Axis I and II disorders in a family study. The American Journal of Psychiatry, 151, 1043-1047.

Kooistra, L., Crawford, S., Dewey, D., Cantell, M., \& Kaplan, B. J. (2005). Motor correlates of ADHD: contribution of reading disability and oppositional defiant disorder. Journal of Learning Disabilities, 38(3), 195-206.

Koppitz, E. M. (1968). Psychological Evaluation of children's human figure drawings. New York, NY: Grune \& Stratton.

Koppitz, E. M. (1971). Children with learning disabilities: A five year follow-up study. New York, NY: Grune \& Stratton.

Koppitz, E. M. (1975) The Bender Gestalt Test for young children, Volume II: Research and application, 1963-1973. New York, NY: Grune \& Stratton.

Krueger, R., \& Finger, M. (2001). Using item response theory to understand comorbidity among anxiety and unipolar mood disorders. Psychological Assessment, 13(1), $140-151$

La Fiosca, T., \& Loyd, B. (1986). Defensiveness and the assessment of parent stress and anxiety in parents. Journal of Clinical Psychology, 15, 254-59.

Lengua, L. J., Sadowski, C. A., Friedrich, W. N., \& Fisher, J. (2001). Rationally and empirically derived dimension of children's symptomology expert ratings and confirmatory factor analyses of the CBCL. Journal of Consulting and Clinical Psychology, 69, 683-698. 
Lipovsky, J. A., Finch, A. J., \& Belter, R.W. (1989). Assessment of depression in adolescence: Objective and projective measures. Journal of Personality Assessment, 53, 449-458.

Luebbe, A. M., Radcliffe, A. M., Callands, T. A., Green, D. \& Thorn, B. E. (2007). Evidence-based practice in psychology: Perceptions of graduate students in scientist-practitioner programs. Journal of Clinical Psychology, 63, 643-655.

McCormick, T. T., \& Brannigan, G. G. (1984). Bender Gestalt Signs as indicants of anxiety, withdrawal, and acting-out behaviors in adolescents. Journal of Psychology, 118(1), 71-74.

McKeon, P. O., Medina, J. M., \& Hertel, J. (2006). Hierarchy of research design in evidenced- based sports medicine. Athletic Therapy Today, 11(4), 41-45.

Mehlman, B., \& Vatovec, E. (1956). A validation study of the Bender-Gestalt. Journal of Consulting Psychology, 20(1), 71-74.

Miyake, A., Friedman, N. P., Rettinger, D. A., Shah, P., \& Hegarty, M. (2001). How are visuospatial working memory, executive functioning, and spatial abilities related? A latent variable analysis. Journal of Experimental Psychology: General, 130, $621-640$.

Morgan, R. D., Olson, K. R., Krueger, R. M., Schellenberg, R. P., \& Jackson, T. (2000). Do the DSM decision trees improve diagnostic ability? Journal of Clinical Psychology 56 (1), 73-88. doi: 10.1002/ (SICI) 1097-4679(200001)56:1<73: AID-JCLP7>3.0.CO; 2-I 
Naglieri, J. A., \& Pfeiffer, S. I. (1992). Performance of disruptive behavior disordered and normal samples on the draw a person: Screening procedure for emotional disturbance. Psychological Assessment, 4(2), 156-159.

Oas, P. (1984). Validity of a Draw-A-Person and Bender-Gestalt as measures of impulsivity with adolescents. Journal of Consulting and Clinical Psychology, 52, 1011-1019.

Pascal, B. J., \& Suttell, G. R. (1952)."Regression" in schizophrenia as determined by performance on the Bender-Gestalt test. The Journal of Abnormal and Social Psychology, 47, 653-657.

Perticone, E. X. (1998). The clinical and projective use of the Bender-Gestalt test. Springfield, IL: Charles C Thomas.

Pilkonis, P. A., Heape, C. L., Ruddy, J., \& Serrao, P. (1991). Validity in the diagnosis of personality disorders: The use of the LEAD standard. Psychological Assessment, $3(1), 46-54$.

Piotrowski, C. (1995). A review of the clinical and research use of the Bender-Gestalt Test. Perceptual and Motor Skills, 81, 1272-1274.

Piotrowski, C., \& Keller, J.W. (1989). Psychological testing in outpatient mental health facilities. Professional Psychology: Research and Practice, 20, 423-425.

Raggi, V. L., \& Chronis, A. M. (2006). Interventions to address the academic impairment of children and adolescents with ADHD. Clinical Child and Family Psychology Review, 9(2), 85-111. doi:10.2007/s10567-006-0006-0 
Reynolds, C. R. (2007). Koppitz developmental scoring system for the Bender-gestalt test-2nd edition (Koppitz 2) Rater's manual. Austin, TX: Pro-Ed Inc.

Roid, G. H. (2003). Stanford-Binet intelligence scales (5th ed.) Itasca, IL: Riverside.

Rossini, E. D. (1983). The Bender-Gestalt psychopathology scale: Failure to infer validity in a school-aged sample. Journal of Personality Assessment, 51, 254-261.

Rossini, E. D. \& Kaspar, J. C. (1987). The validity of the Bender- Gestalt emotional indicators. Journal of Personality Assessment, 51, 254-261.

Shapiro, S. K., \& Simpson, R. G. (1995). Koppitz Scoring System as a measure of Bender -Gestalt performance in behaviorally and emotionally disturbed adolescents. Journal of Clinical Psychology, 51(1), 108-112.

Smith, D. J., Muir, W. J., \& Blackwood, D. H. (2006). Neurocognitive impairment in euthymic young adults with bipolar spectrum disorder and recurrent major depressive disorder. Bipolar Disorder, 8(1), 40-46.

Sourander, A., Haavisto, A., Ronning, J.A., Multimaki, P. Parkkola, K., Santalahti, P., ... Almqvist, F. (2005). Recognition of psychiatric disorders, and self perceived problems. A follow up study from age 8 to age 18. Journal of Child Psychology and Psychiatry, 46, 1124-1134.

Spitzer, R. L. (1983). Psychiatric diagnosis: Are clinicians still necessary? Comprehensive Psychiatry, 24, 399-411.

Stewart, H. F. (1957). A note on recall patterns using the Bender Gestalt with psychotic and non-psychotic patients. Journal of Clinical Psychology, 13, 95-97. 
Tolan, P. H., \& Dodge, K. A. (2005). Children's mental health as a primary care and concern: A system for comprehensive support and service. American Psychologist, 60, 601-614.

Valderhaug, R., \& Ivansson, T. (2005). Functional impairment in clinical samples of Norwegian and Swedish children and adolescents with obsessive-compulsive disorder. European Child \& Adolescent Psychiatry, 14(3), 164-173.

Wassenberg, R., Max, J. E., Koele, S. L., \& Firme, K. (2004). Classifying psychiatric disorders after traumatic brain injury and orthopedic injury in children; adequacy of KSADS versus CBCL. Brain Injury, 18, 377-390. doi:10.1080/ 02699250310001617325

Wertheimer, M. (1923). Laws of organization in perceptual forms. First published as Untersuchungen zur Lehre von der Gestalt II, in Psycologische Forschung, 4, 301-350. Translation published in Ellis, W. (1938). A source book of Gestalt psychology (pp. 71-88). London: Routledge \& Kegan Paul.

Wilson, M. S., \& Reschly, D. J. (1996). Assessment in school psychology training and practice. School Psychology Review, 25(1), 9-23.

Woodcock, R. W., McGrew, K. S., \& Mather, N. (2001). Woodcock Johnson III Tests of Achievement. Itasca, IL: Riverside.

Youngstrom, E. (2005). Improving the assessment of juvenile bipolar disorder. Unpublished Training Manual. (NIH R01 5MH066647) 
Youngstrom, E. A. (2006) Training manual for $A B A C B$. Unpublished work for Applewood Centers, Cleveland, $\mathrm{OH}$.

Youngstrom, E. A., \& Duaz, B.A. (2005). Evidenced based assessment of Pediatric Bipolar Disorder, Part I: Base rate and family history. Journal of American Academy of Adolescent Psychiatry, 44, 712-717.

Youngstrom, E. A., Findling, R. L., Danielson, C. K., \& Calabrese, J. R. (2001).

Discriminative validity of parent report of hypomania and depressive symptoms of the General Behavior Inventory for juvenile bipolarity. Psychological Assessment, 13, 267-276.

Youngstrom, E. A., Loeber, R., \& Stouthamer-Loeber, M. (2000). Patterns and correlates of agreement between parent, teacher, and male adolescent ratings of externalizing and internalizing problems. Journal of Consulting and Clinical Psychology, 68, 1038-1050.

Youngstrom, E. A., Meyers, O., Demeter, C., Youngstrom, J., Morello, L., Piiparinen, R..., \& Findling, R. L. (2005). Comparing diagnostic checklists for pediatric bipolar disorder in academic and community mental health setting. Bipolar Disorders 7, 507-517.

Zweig, M. H., \& Campbell, G. (1993). Receiver operating characteristic (ROC) plots: a fundamental evaluation tool in clinical medicine. Clinical Chemistry 39, 561-577. 


\section{Appendix A: IRB Protocol}

University Hospitals of Cleveland

Case Western Reserve University IRB Number 01-02-39

Title: Improving the Assessment Process of Children 
Proposed IRB Addendum (2007)

\section{Overview:}

The proposed amendment would add one measure of developmental visual motor ability to the ongoing study. The addition of this measure would not increase the length of the visit for the participating families at all. This was because the parent interview portion takes substantially longer than the youth interview component. As a result the addition of the measure filled a gap when the youth would otherwise be waiting for the parent to complete their portion of the assessment. The introduction of this measure would provide valuable information regarding the individual functioning of the child and would form the basis of a doctoral dissertation comparing the KSADS-PL with the Bender Gestalt Visual Motor Integration Test using two newly reintroduced results. Thus the addition would add greatly to the validation of clinically driven measurements, contribute to a limited body of knowledge regarding these measures, and as well have the potential to fulfill important educational goals.

\section{Rationale:}

Using a group of participants already being measured using approved research methods and deidentified data, a secondary analysis would be performed incorporating the Bender Visual Motor Gestalt Test II (Brannigan \& Decker, 2006). The Bender Gestalt II is a revision of the original Bender gestalt test developed by Bender in 1938 (Bender, 
1938), which had been one of the most used measures in psychological assessments until the 1990's (Archer, Maruish, Imhof, \& Piotrowski, 1991; Brannigan \& Decker, 2006). Its clinical attractiveness was that it was quick to give, taking less than 10 minutes, was used in an ice breaker in most psychological evaluations because of its ease on the subject, found to be a cross cultural, nonverbal measure that had a scoring capabilities for a large age range (Brannigan \& Decker, 2006). The Bender fell out of favor with many psychologists due to research cautioning the broad use of the Bender for diagnostic evaluations (Bigler \& Ehrfurth, 1981) and low reliability with regard to its use for projective means (Naglieri \& Pfeiffer, 1992).

The reality is that clinicians were in want of effective diagnostic instruments like the KSADS-PL yet have to manage the time/ cost balance in practice, this study allows the unique opportunity of comparing the utility of the Bender Gestalt II as a screening instrument with regard to diagnostic criteria. The study allows the unique opportunity of a comparison of the clinically efficient measure of the Bender with the diagnostic reliability of the KSADS-PL. Completing the Bender Gestalt-II measure is not difficult for the client and typically takes less than 15 minutes total time for the participant, and would not add any time to the total amount that families spend participating in the current study. Individual functioning of children is often evaluated to determine level of current functioning/ability in paper and pencil tasks such as the BVMGT-II. Over the years, such testing instruments have been compared to various constructs such as achievement levels, perceptual ability, visual motor ability, developmental capability and various emotional 
symptoms related to various diagnostic categories (Bender, 1938; Decker, Allen, \& Choca, 2006; Hutt, 1985; Valderhaug \& Ivansson, 2005). 


\title{
IRB Protocol
}

WestVirginiaUniversity.

Office of Research Compliance

\section{Expedited - IRB Protocol - Non Human Subjects Research}

\author{
To: $\quad$ Tunick, Roy \\ From: $\quad$ WVU Office of Research Compliance \\ Date: $\quad$ Monday, March 24, 2008 \\ Subject: No action required
}

Tracking \#: H-20822

Title: The Clinical utility of the Bender Gestalt II and the Koppitz 2 compared with the KSADS-PL+ in children 5 to 18.

Thank you for your submission to the West Virginia University [boardname] Institutional Review Board. On [Review Date Not Found], the submission was reviewed. As described, it was determined that your project does not constitute human subject research, per the 10 August 2004 Office for Human Research Protections Guidance on Research Involving Coded Private Information or Biological Specimens. Please contact the IRB office for a copy of the guidance.

Furthermore, it was determined that:

In discussions with the $\mathrm{PI}$, it is clear that the data to be analyzed will be deidentified before the $\mathrm{PI}$ receives it. There is no way for the PI to match a name to the data and therefore no identifiable individual. The study is therefore not human subject research. If you have any questions, please contact the IRB at (304) 293-7073.

Thank you.

Board Designee: Ast, Lilo

Letter Sent By: Ast, Lilo, 3/24/2008 6:03 PM 


\section{Appendix B: CREC Program Notice of Certification}

\section{Linda Marnic,}

Congratulations! You are Core Certified in the Continuing Research Education Credit (CREC) Program managed by Case Western Reserve University (Case). To obtain Core certification you passed the online CITI Core Training course. Core Certification means that you would have the ability to request review of proposed human subject research proposals by Institutional Review Boards at the following institutions: University Hospitals of Cleveland, The Metro Health System, and Case Western Reserve University.

Certification in the CREC Program also means that you have met the NIH educational requirements for the involvement of human participants in research for Key Personnel. This certification is valid for 3 years and would expire on 11/15/2009. To be Re-Certified after this date you must obtain 12 CRECs before your Core Certification expires. The following is the URL to our educational web site outlining the training options available for continuing certification as well as provide instructions for obtaining information on your current CREC status by looking up your account information on SPRIDERWEB, Sponsored Projects Information and Data Entry and Retrieval Website.

\section{http://ora.ra.cwru.edu/orc_education.asp}

You may view a printable "Certificate of Achievement" by going here. Please maintain this document for your records. Copies of this document may also be submitted to sponsors to indicate compliance with human subject education requirements.

I hope this information is helpful. Please feel free to contact me if I can be of further assistance.

The CREC Program

Sears 657, CWRU

216.368.6925

CREC@case.edu 


\title{
Appendix C: Administration Manual for ABACAB
}

\author{
Bender Gestalt II Administration Manual for $A B A C A B$ \\ I. Who Administers the BG II?
}

Either the Rater 1 or the Rater 2 with appropriate training would administer the test. It would be decided on a team basis by the raters.

Suggested time of testing: If Rater 2 administers the standard protocol, and then the BG II should be administered prior to the questionnaires. If Rater 1 administers the protocol, then the BG II should be administered at the end the KSADS if time permits.

If the family is finished before 3:30 pm, the BG II must be administered before finalizing with the family.

\section{What is needed to administer the BG II?}

The BG II stimulus cards, BG II observation form (ABACAB version), a motor test, a perception test, 2 pencils with erasers, 10 sheets of blank printer paper, and a time keeping device (stopwatch or a watch or clock that had seconds).

\section{What is the administration order?}

The official and only acceptable administration order is: copy, recall, motor, and perception. Each phase occurs immediately after the previous phase. There are no breaks between phases, unless absolutely necessary (this should be marked).

\section{How do I administer the Copy test?}

Place the cards in order; design side down. Place a blank sheet of paper vertically and a pencil in front of the child. Read the following directions:

I have a number of cards here. Each card has a different drawing on it. I would show you the cards one at a time. Use this pencil to copy the drawing from each

card into this sheet of paper. Try to make your drawings look just like the drawings on the cards. There are no time limits, so take as much time as you need. Do you have any questions? Here is the first card.

Show the child the first card and mark the starting time (include seconds). Administer cards 1 through 13 in order to children below age 8. Administer cards 5 through 16 in order to children above age 8. Children may erase and use more than 1 sheet of paper. Do not allow the children to touch the stimulus cards or draw/doodle nontest figures on the paper.

If the examinee becomes discouraged say: 


\section{Do the best you can.}

If the examinee asks where to start drawing any figure, say:

Begin wherever you like.

When complete, record the time finished (include seconds). Label the child's sheet: COPY SHEET and TOP on the top of the page as started on by the child.

Things to be marked on the Observation sheet by the administrator:

1) Direction and order of drawing

2) Describe any counting that occurs

3) If any of the test-taking observations occur on more than 2 items, check the box.

4) Mark the tilt of the paper.

\section{How do I administer the Recall Phase?}

Immediately after the Copy phase, give the child a new sheet of paper placed vertically. Read:

Now, I want you to draw as many of the designs that I just showed you as you can remember. Draw them on this new sheet of paper. Try to make your drawings just like the ones on the cards that you saw earlier. There are no time limits, so take as much as you need. Do you have any questions? Begin.

Begin timing (include seconds). Stop timing when child finishes all figures or does not recall any more designs after 2 minutes. Record end time (include seconds). Label the top of the sheet: Recall Sheet and Top (for top of sheet from child's beginning).

On the Observation sheet, mark the order in which the items were recalled.

\section{How do I administer the Motor Test?}

Immediately after the recall phase, hand the child the BG II Motor Test. Say:

For each item, start with the largest figure. For each figure draw a line connecting the dots without touching the borders. Do not lift the pencil, erase, or tilt the paper while drawing. Try the sample item. Do you have any questions? Now you try it.

Make sure the child completes all items. Repeat directions as needed. 


\section{How do I administer the Perception Test?}

Immediately after the Motor Test, hand the child the BG II Perception Test. Say:

Look at this picture (point to the design in the first box). There is another picture that looks just like it in this row (run finger across the first row). Circle or point to the picture that looks just like this one (point at design in first box).

If the child needs assistance for any item say:

\section{Which one of these pictures looks like this one? (Point at picture in first box of row)}

If it takes the child more than 30 seconds, then say:

Let's try the next one. Write an $S$ next to any skipped items.

\section{How do I score the BG II Copy and Recall Phases?}

The scoring system is $0-4$ (no resemblance to nearly perfect). Please score according to the following pictures for each item. If uncertain refer to Bender Gestalt Manual for further pictures.

**Instructions for this manual were from the Bender Gestalt II Manual (2006). 


\section{Demographic Forms}

CONTACT INFORMATION Subject \#:
Child's Name First

Middle

Last

Nickname:

Child's Date of Birth: -

Address:

Parent/Guardian's

Name:

Relationship of guardian to child:

Address:

(if different from children) Street/Apt\#

Zip

City

State

Phone: ( ) - __ Work Phone: ( )

Cell Phone: (

$\longrightarrow$ - ___ User of Cell:

E-mail address:

If applicable, other parent/guardian's name:

Relationship of other parent/ guardian to child:

Other parent/guardian's home address:

City

State

Zip

Home telephone (if different): ( )

Work Phone: (

Cell Phone: (

)

$-$

User of Cell:

Emergency contact person: 
Relation (if known):

Phone: ( )

Alt. Emergency contact person:

Relation (if known):

Phone: (

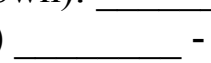

Date of Assessment:

Rater 2 Interview with Primary Caregiver

ACI ID

Date of Review

Employ these codes on the pages that follow: 





Appendix E: Consent Forms 
UNTVERSTY HOSPITALS OF CLEVELAND CONSENT FOR INVESTIGATIONAL STUDIES

Project Title: Improving the Assessment of Juvenile Psychiatric Disor ders

Principal Investigators: Eric A. Youngstrom, $\mathrm{Ph} . \mathrm{D}$, Oren I Meyers, $\mathrm{Ph} \mathrm{D}$

Consent

L/my child am being asked to participate in a questionnaire study to determine if I/my child may have an emotional or behavior problem. The pupose of this study is to carefully assess the emotional/behavioral difficulties I/my child may have $\mathrm{I} / \mathrm{my}$ child understand that it may be determined that $\mathrm{J}$ my child does not have a behavior or emotional problem.

\section{Study Participation}

As part of this study, Lmy child will be asked a series of questions. We will be asked to complete questionnaires about L/my child's emotional states and behaviors. Both $\mathrm{I} / \mathrm{my}$ child will be interviewed and will complete questionnaires. A member of the research group may ask that one of my/my child's teachers complete several questionnaires about my/my child's emotions, learning and behaviors. If $I / m y$ child sign a written release of information allowing them to contact my/my child's wulpıetc questonnaites aoout v/my cnula's emotional states and behaviors. Both $\mathrm{I} / \mathrm{my}$ child will be interviewed and will complete questionnaires. A member of the research group may ask that one of my/my child's teachers complete several questionnaires about my/my child's emotions, learning and behaviors. If $\mathrm{I} / \mathrm{my}$ child sign a written release of information allowing them to contact my/my child's teacher, a member of the research group will contact my/my child's teacher in order to explain the questionnaires to them. All these procedures generally take approximately 4-5 hours to complete. If I/my child am being seen at University Hospitals, I/my child may be asked to return for 3 follow-up visits at six-month intervals to complete questionnaircs about my/my child's emotional states and behaviors. These follow-up visits will take approximately 1.5 hours. If $1 /$ my child am being seen at University Hospitals, then I/my child may be referred to an associated child psychiatrist ot psychologist for further evaluation. This appointment may depend on whether or not I/my child am interested in joining a treatment program being offered by the Stanley Research Center at University Hospitals of Cleveland While I/my child am being asked questions, the session may be audio taped in order for the study staff to be able to review the ariswers at a later time. These tapes will be kept in a locked closet and will be destroyed using a giant magnet to erase its contents, when $\mathrm{L}$ my child complete the study.

\section{Risks and Discomfor ts}

One of the risks associated with this studv is that the auestions asked to me/mv child mav he 
the study.

\section{Risks and Discomforts}

One of the risks associated with this study is that the questions asked to me/my child may be upsetting to me/my child.

CONSENT FORM APPROVED FOR USE WITH PROTOCOL: 으:-02-34 EXPIRATIONDATE $1 / 15 / 2008$

\section{UNIVERSITY HOSPITALS OF CLEVELAND CONSENT FOR INVESTIGATIONAL STUDIES}

Project Title: Improving the Assessment of Juvenile Psychiatric Disorders

Principal Investigators: Eric A. Youngstrom, $\mathrm{Ph} D$, Oren I. Meyers, $\mathrm{Ph} \mathrm{D}$

Consent

If $\mathrm{I} / \mathrm{m}$ y child do not wish to answer a question, $\mathrm{V} / \mathrm{my}$ child may skip it and go to the next question

Another risk of this study is that $\mathrm{I}$ my child may become fatigued from answering questions asked of me/my child L/my child may stop at anytime without consequence. If during the course of the interview I/my child am found to be at suicidal or homicidal risk, an appropriate member of the study team will be notified. This notification will take place in order to keep me/my child, and others safe from harm. Additionally, if any incidents of abuse are disclosed during the course of the interview, the appropriate authorities will be notified if a report has not previously been filed.

\section{Potential Benefits of the Study}

A possible bencfit to participating in this study is the opportunity to meet with a research clinician and IF I/MY CHILD AM BEING SEEN AT UNIVERSITY HOSPITAL AS PART OF 


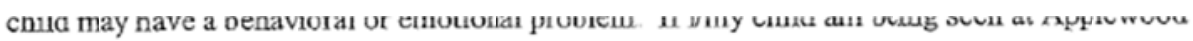
Centers, Inc., V/my child may not have the opportunity to see a child psychiatrist pertaining to this study. Vmy child's participation in this study may aid in the understanding of how to describe the problems some kids and/or their parents may have with their feelings and the way they behave.

\section{Compensation for Participation}

I/my child will receive $\$ 40$ (forty dollars) for the first visit if all study measures are completed regarding me/my child If I/my child am being seen at University Hospitals, I/my child may be asked to return for three additional visits and will receive $\$ 40$ (forty dollars) and a gift certificate for a video rental of $\$ 10$ for each additional visit if all study measures are completed regarding me/my child. If I/my child am being seen at University Hospitals and I/my child am asked to return for three additional visits, then the total possible amount of compensation for participation is $\$ 160$ for completion of the entire protocol and follow up visits If I/my child am being seen at Applewood Version date: $6 / 28 / 2006$ Page 2 of 5

\section{UNIVERSITY HOSPITALS OF CLEVELAND CONSENT FOR INVESTIGATIONAL STUDIES}

Project Title: Improving the Assessment of Juvenile Psychiatric Disorders

Principal Investigators: Eric A Youngstrom, $\mathrm{Ph}$. D., Oren I. Meyers, $\mathrm{Ph}$. D

Consent

Centers, Inc, I/my child's participation in this study will be complete at the end of the interview today and $\mathrm{V}$ my child will not be asked to return for three additional visits. I agree to be recontacted in the future if $\mathrm{V}$ my child qualify for participation in a future study

\section{Results of Assessments}

I/my child will not receive a copy of the completed questionnaires, nor will $\mathrm{I} /$ my child receive a written report In addition, $\mathrm{V} /$ my child may not receive a working official diagnosis regarding my/my child's condition. However, I/my child can request verbal feedback about the results. I/my child may have a written summary released to a health professional of our choice after I sign a written release of information form 
Clinical care is not provided as part of this study If $\mathrm{L} / \mathrm{my}$ child refuse to participate in this study, and I/my child am concemed about my/my child's behaviors or emotions, I/my child should seek care for myselfimy child with a mental health provider of my/my child's choosing. If $I / m y$ child refuse to participate in this study, this will in no way jeopardize my/my child's current or future care at University Hospitals of Cleveland or Applewood Centers, Inc

\section{Alternatives to Participation}

If $I / m y$ child choose not to participate in this study and $\mathrm{I} / \mathrm{my}$ child am concerned about $\mathrm{I} / \mathrm{m} y$ child's behaviors or emotions, then L/my child can seek care with another mental health provider of my/my child's choice (for example a psychologist, physician, social worker, etc ) at another facility

\section{Summary of Your Rights as a Participant in a Research Study}

V/my child's participation in this research study is voluntary. Refusing to participate will not alter my/my child's usual health carc or involve any penalty or loss of benefits to which I/my child am otherwise entitled If $\mathrm{l}$ my child decide to join the study, I/my child may withdraw at any time and for Version date: $6 / 28 / 2006$ Page 3 of 5 


\section{Appendix F: Statistical Findings}

Statistical findings /SPSS 16.0

Sample Demographics.

\begin{tabular}{cc} 
Age, years & \\
Median & 12 \\
Mean & 11.92 \\
SD & 2.56 \\
Minimum-Maximum & $8-17$ \\
Number of Diagnoses & \\
Median & 3 \\
Mean & 2.76 \\
SD & 1.42 \\
Minimum-Maximum & $0-8$ \\
Gender, n $\%)$ & \\
Boy & $48(64)$ \\
Girl & $27(36)$ \\
Ethnicity, n (\%) & \\
White & $83(84)$ \\
Black & $4(5)$ \\
Other & $61)$ \\
\hline
\end{tabular}




\begin{abstract}
Author's Note
This work is in completion of a Ph.D. dissertation for Linda R. Marnic, Department of Counseling Psychology at West Virginia University. Professional colleagues that were integral to this work include Eric Youngstrom, Department of Psychology, University of North Carolina at Chapel Hill; Oren Meyers, Department of Psychiatry, Case Western Reserve University, Cleveland, Ohio; Andrew Freeman, University of North Carolina at Chapel Hill; Heather Marcinek, Case Western Reserve University; Frank Ezzo, Department of Psychology, Applewood Centers, Cleveland, Ohio.

The research was supported in part by a grant funded by the National Institute of Mental Health in coordination with a study of Bipolar Disorder in Children.

Correspondence regarding this research may be sent to Linda R. Marnic, who is now in private practice at Family Matters, P.O. Box 490, Lost Creek, West Virginia 26385

E-mail: 1marnic@wvfamilymatters.com
\end{abstract}


Virginia University Libraries, ou=Acquisitions Department, Hagen $\begin{aligned} & \text { email=John.Hagen@mail.wvu.edu, } \\ & \text { c=Us } \\ & \text { Date: 2010.12.08 15:41:13-05'00' }\end{aligned}$ 